Article

\title{
Regular Ordered Ternary Semigroups in Terms of Bipolar Fuzzy Ideals
}

\author{
Shahida Bashir ${ }^{1, *}$, Medhit Fatima ${ }^{1}$ and Muhammad Shabir ${ }^{2}$ \\ 1 Department of Mathematics, University of Gujrat, Gujrat 50700, Pakistan; medhit.fatima6@gmail.com \\ 2 Department of Mathematics, Quaid-i-Azam University, Islamabad 44000, Pakistan; \\ mshabirbhatti@yahoo.co.uk \\ * Correspondence: shahida.bashir81@gmail.com or shahida.bashir@uog.edu.pk
}

Received: 25 December 2018; Accepted: 26 February 2019; Published: 4 March 2019

\begin{abstract}
Our main objective is to introduce the innovative concept of $(\alpha, \beta)$-bipolar fuzzy ideals and $(\alpha, \beta)$-bipolar fuzzy generalized bi-ideals in ordered ternary semigroups by using the idea of belongingness and quasi-coincidence of an ordered bipolar fuzzy point with a bipolar fuzzy set. In this research, we have proved that if a bipolar fuzzy set $h=\left(S ; h_{n}, h_{p}\right)$ in an ordered ternary semigroup $S$ is the $(\in, \in \vee q)$-bipolar fuzzy generalized bi-ideal of $S$, it satisfies two particular conditions but the reverse does not hold in general. We have studied the regular ordered ternary semigroups by using the $(\in, \in \vee q)$-bipolar fuzzy left (resp. right, lateral and two-sided) ideals and the $(\in, \in \vee q)$-bipolar fuzzy generalized bi-ideals.
\end{abstract}

Keywords: ordered ternary semigroup; bipolar fuzzy ideal; bipolar fuzzy generalized bi-ideal; $(\alpha, \beta)$-bipolar fuzzy generalized bi-ideal

\section{Introduction}

In real life, the bipolar fuzzy set theory is a core feature that should be considered. This theory distinguishes between the positive information and negative information for the same object when they have the same characteristics. For example, a house close to the commercial area of a city is both good (it is convenient) and bad (it is noisy). Similarly, $\$ 100$ represents significantly more for a poor man but at the same time, this $\$ 100$ may have less value for a millionaire. Zadeh [1] has provided a strong mathematical tool to deal with complex and uncertain behaviors. However, the membership degrees of the fuzzy sets are limited in the interval $[0,1]$. This makes it difficult to express the diversity of the information of the fuzzy set. In the solution of the above-mentioned flaw, Zhang in [2] introduced the idea of bipolar fuzziness as an extension of the fuzzy set in which the membership degree range is $[-1,1]$.

The traditional fuzzy set representation cannot distinguish contrary elements from irrelevant elements. For example, consider a fuzzy set "young" defined in the age domain [0, 100]. Furthermore, consider two ages of 50 and 95 with a membership degree of 0 . Although both of them do not satisfy the property "young", we may say that the age 95 is more separate from the property rather than age 50 . When the membership degree is in the interval range $[0,1]$, it is difficult to express the difference between the irrelevant elements and the contrary elements in the fuzzy sets. If a set representation could express this type of information, it would be more informative than the traditional fuzzy set. This type of fuzzy set is called the bipolar fuzzy set. The results and examples related to the bipolar fuzzy sets bipolar fuzzy finite state machine are shown in [3-6].

There are some structures that are not appropriately handled when using the binary operation of the semigroup. For example, in the set $\mathrm{Z}$ of integers, its subset $\mathrm{Z}^{+}$is closed with respect to the binary operation and the ternary operation. However, if we take only $Z^{-}$the set of negative integers, it is not 
closed with respect to the binary operation but remains closed with respect to the ternary operation. To deal with this type of problem, we studied the ordered ternary semigroup.

Fuzzy semigroups were generalized twice, which namely produced the fuzzy ordered semigroups and fuzzy ternary semigroups. Ordered semigroups are useful for computer science. In particular, in the theory of automata and formal language, fuzzy ordered semigroups has been extensively studied (see [7-11]). Different types of regularity of ordered semigroups and ternary semigroups are characterized in terms of fuzzy ideals, such as weakly regular (or quasi-regular) ordered semigroups [12-16]. In [16], Shabir et al. provided the characterizations of regular semigroups by $(\alpha, \beta)$-fuzzy ideals. and regular ternary semigroups $[17,18]$. Since the bipolar fuzzy theory is an extension of the fuzzy theory, one may expect that the bipolar fuzzy ordered ternary semigroups will be useful in studying the bipolar fuzzy automata theory and the bipolar fuzzy formal language.

Lee [19] presented the notion of bipolar fuzzy ideals of BCK $\backslash$ BCI-algebras. Ibrar, Khan and Davvaz [20] used $(\alpha, \beta)$-bipolar fuzzy generalized bi-ideals for the characterizations of the regular ordered semigroups. In this paper, we have proved that a set of bipolar fuzzy subsets in an ordered ternary semigroup is also an ordered ternary semigroup. Before this paper, this theorem was just used for ordered semigroups in [20]. Similarly, we have outlined our next major contribution of this paper. We have proved that if a bipolar fuzzy set in an ordered ternary semigroup is a $(\in, \in \vee q)$-bipolar fuzzy generalized bi-ideal of $S$, it satisfies two particular properties of $S$. However, we have shown with an example that the reverse of this statement is not true in general. This result has negated the result proved in [20].

We have characterized the regular ordered ternary semigroups in terms of the bipolar fuzzy ideals and $(\alpha, \beta)$-bipolar fuzzy generalized bi-ideals. Several results in $[20,21]$ are generalized and new results are found.

\section{Preliminaries}

A non-empty partially ordered set $(S, \leq)$ with a ternary operation is said to be an ordered ternary groupoid $(S, \cdot, \leq)$ if the ternary operation is represented by the juxtaposition and partial ordering $\leq$ with $p_{1} p_{2} p_{3} \leq q_{1} q_{2} q_{3}$ for any $p_{i}, q_{i} \in S$ and $p_{i} \leq q_{i}, i=1,2,3$ or equivalently, $p_{1} \leq p_{2}$ shows that $p_{1} p_{3} p_{4} \leq p_{2} p_{3} p_{4}, p_{3} p_{4} p_{1} \leq p_{3} p_{4} p_{2}$ for all $p_{1}, p_{2}, p_{3}, p_{4} \in S$. Moreover, if we assume that $(S, \cdot)$ is a ternary semigroup, an ordered ternary semigroup can be denoted as $(S, \cdot, \leq)$.

One can perceive the ternary semigroup as an ordered ternary semigroup with insignificant ordering. A natural phenomenon shows that the ternary operations are followed by the binary operations in the ordered ternary semigroups. In this paper, subsets mean non-empty subsets. For any subset $F$ containing an ordered ternary groupoid $S, F^{3}$ will be written as $F F F=\{a b c: a, b, c \in F\}$.

If we consider $S$ as an ordered ternary groupoid and $F \subseteq S$, we know that $(F]=\{h \in S \mid h \leq l$ for any $l \in F\}$. In the same way, if $F, G$ and $H$ are subsets of an ordered ternary groupoid and if $F \subseteq G$, we know that $(F] \subseteq(G]$ and $((F](G](H]) \subseteq(F G H]$. Any subset $F$ of an ordered ternary groupoid $S$ will be known as the left (resp. lateral, right) ideal of an ordered ternary semigroup, assuming that $(F]=F$ and $S S F \subseteq F$ (resp. $S F S \subseteq F, F S S \subseteq F)$. Any left, lateral and right ideal is termed as an ideal. Any left and right ideal is termed as a two-sided ideal [21].

A subset $F$ of an ordered ternary semigroup is known as the generalized bi-ideal of $S$ provided that $(F]=F$ and $F S F S F \subseteq F$. A subset $F$ of an ordered ternary semigroup is called the subsemigroup of $S$ if $(F]=F$ and $F^{3} \subseteq F$. Any subsemigroup $F$ of an ordered ternary semigroup is known as the bi-ideal of $S$ if $(F]=F$ and $F S F S F \subseteq F$ [22]. Each bi-ideal of an ordered ternary semigroup $S$ is a generalized bi-ideal of $S$ but its reverse does not hold generally. 
Example 1. Let $S=\{\alpha, \beta, \gamma, \delta\}$ with $\alpha \beta \gamma=\alpha(\beta \gamma)$ be an ordered ternary semigroup with the following multiplication table and order below:

$\begin{array}{lllll}\cdot & \alpha & \beta & \gamma & \delta \\ \alpha & \alpha & \alpha & \alpha & \alpha \\ \beta & \alpha & \alpha & \alpha & \alpha \\ \gamma & \alpha & \alpha & \beta & \alpha \\ \delta & \alpha & \alpha & \beta & \beta\end{array}$

$\leq=\{(\alpha, \alpha),(\beta, \beta),(\gamma, \gamma),(\delta, \delta),(\alpha, \beta)\}$. The subsemigroups of $S$ are presented as $\{\alpha\},\{\alpha, \beta\},\{\alpha, \beta, \gamma\},\{\alpha, \beta, \delta\}$ and $\{\alpha, \beta, \gamma, \delta\}$. All the subsemigroups of $S$ are bi-ideals. The generalized bi-ideals of $S$ are $\{\alpha\},\{\alpha, \beta\},\{\alpha, \gamma\},\{\alpha, \delta\},\{\alpha, \beta, \gamma\},\{\alpha, \beta, \delta\},\{\alpha, \gamma, \delta\}$ and $\{\alpha, \beta, \gamma, \delta\}$. However, on the other hand, $\{\alpha, \gamma\},\{\alpha, \delta\}$ and $\{\alpha, \gamma, \delta\}$ are not bi-ideals of $S$ because they are not subsemigroups of $S$.

A bipolar-valued fuzzy set $h$ of an ordered ternary groupoid $S$ has the form of $h=\left(S ; h_{n}, h_{p}\right)$ where $h_{n}: S \rightarrow[-1,0]$ and $h_{p}: S \rightarrow[0,1]$ are mappings. $h_{n}(m)$ represents the satisfaction degree of an object $m$ to the counter property of $h=\left(S ; h_{n}, h_{p}\right)$ and $h_{p}(m)$ represents the satisfaction degree of an element $m$ to the corresponding property of $h=\left(S ; h_{n}, h_{p}\right)$.

If $h_{p}(m)=0$ and $h_{n}(m) \neq 0$, this means that $m$ does not satisfy the property of $h$. If $h_{p}(m) \neq 0$ and $h_{n}(m)=0$, this situation means that $m$ only considered the positive satisfaction for $h=\left(S ; h_{n}, h_{p}\right)$. This is also possible for an element $m$ with $h_{n}(m) \neq 0$ and $h_{p}(m) \neq 0$. This happens when a membership function of the property intersects with some portion of the domain. For the bipolar-valued fuzzy set, $h=\left(S ; h_{n}, h_{p}\right)$. Furthermore, we will use the terminology of the bipolar fuzzy sets instead of using the bipolar-valued fuzzy sets.

$B F(S)$ represents the collection of all bipolar fuzzy subsets of the ordered ternary semigroup $S$. We also express an ordered relation $\leq$ on $B F(S)$ in this way. If $h=\left(S ; h_{n}, h_{p}\right)$ and $g=\left(S ; g_{n}, g_{p}\right)$ are two bipolar fuzzy sets in $S, h \leq g$ if and only if (for every $m \in S) h_{n}(m) \geq g_{n}(m)$ and $h_{p}(m) \leq g_{p}(m), h=g$ if and only if $g \leq h$ and $h \leq g$. Furthermore, $h \cap g=\left(S ; h_{n} \vee g_{n}, h_{p} \wedge g_{p}\right), h \cup g=\left(S ; h_{n} \wedge g_{n}, h_{p} \vee g_{p}\right)$. For any ordered ternary groupoid $S$, the bipolar fuzzy sets $0=\left(S ; 0_{n}, 0_{p}\right)$ and $1=\left(S ; 1_{n}, 1_{p}\right)$ are illustrated as (for all $m \in S) 0_{n}(m)=0=0_{p}(m), 1_{n}(m)=-1$ and $1_{p}(m)=1$. This is the usual practice to examine that $(B F(S), \leq)$ is a poset that has 0 as a minimum element and 1 as the maximum element. We can state that $a \in S$ :

$$
A_{a}=\{(l, m, n) \in S \times S \times S \mid a \leq l m n\} .
$$

Considering three bipolar fuzzy sets $h=\left(S ; h_{n}, h_{p}\right), g=\left(S ; g_{n}, g_{p}\right)$ and $f=\left(S ; f_{n}, f_{p}\right)$ of $S$, we define the product as $h \circ g \circ f=\left(S ; h_{n} \circ g_{n} \circ f_{n}, h_{p} \circ g_{p} \circ f_{p}\right)$ where

$$
\begin{aligned}
& \left(h_{n} \circ g_{n} \circ f_{n}\right)(a)=\left\{\begin{array}{cc}
\wedge\left\{h_{n}(l) \vee g_{n}(m) \vee f_{n}(n)\right\} & \text { if } A_{a} \neq \varphi \\
0 & \text { if } A_{a}=\varphi
\end{array}\right. \\
& \left(h_{p} \circ g_{p} \circ f_{p}\right)(a)=\left\{\begin{array}{cc}
\vee\left\{h_{p}(l) \wedge g_{p}(m) \wedge f_{p}(n)\right\} & \text { if } A_{a} \neq \varphi \\
0 & \text { if } A_{a}=\varphi .
\end{array}\right.
\end{aligned}
$$

Clearly, it can be seen that the multiplication $\circ$ on $B F(S)$ is well-defined and $(B F(S), 0)$ is a ternary groupoid with zero $0=\left(S ; 0_{n}, 0_{p}\right)$. That is, $0_{p} \circ h_{p} \circ g_{p}=h_{p} \circ 0_{p} \circ g_{p}=h_{p} \circ g_{p} \circ 0_{p}=h_{p} \circ 0_{p} \circ 0_{p}=$ $0_{p} \circ g_{p} \circ 0_{p}=0_{p} \circ 0_{p} \circ f_{p}=0$ and $0_{n} \circ h_{n} \circ g_{n}=h_{n} \circ 0_{n} \circ g_{n}=h_{n} \circ g_{n} \circ 0_{n}=h_{n} \circ 0_{n} \circ 0_{n}=$ $0_{n} \circ g_{n} \circ 0_{n}=0_{n} \circ 0_{n} \circ f_{n}=0$ for every $h_{,} g, f \in B F(S)$. Clearly, for $h_{i}, g_{i}, f_{i} \in B F(S), i=1,2,3$, we have $h_{1} \circ h_{2} \circ h_{3} \leq g_{1} \circ g_{2} \circ g_{3}$ if and only if $\left(h_{1 n} \circ h_{2 n} \circ h_{3 n}\right)(a) \geq\left(g_{1 n} \circ g_{2 n} \circ g_{3 n}\right)(a)$ and $\left(h_{1 p} \circ\right.$ $\left.h_{2 p} \circ h_{3 p}\right)(a) \leq\left(g_{1 p} \circ g_{2 p} \circ g_{3 p}\right)(a)$ for any $a \in S$ with $A_{a} \neq \varphi$. Particularly, $h_{1} \circ h_{2} \circ h_{3}=g_{1} \circ g_{2} \circ g_{3}$ if and only if $\left(h_{1 n} \circ h_{2 n} \circ h_{3 n}\right)(a)=\left(g_{1 n} \circ g_{2 n} \circ g_{3 n}\right)(a)$ and $\left(h_{1 p} \circ h_{2 p} \circ h_{3 p}\right)(a)=\left(g_{1} \circ g_{2 p} \circ g_{3 p}\right)(a)$ for any $a \in S$ with $A_{a} \neq \varphi$. 
Definition 1. A bipolar fuzzy set $h=\left(S ; h_{n}, h_{p}\right)$ of an ordered ternary groupoid $S$ is called the fuzzy left (resp. lateral, right) ideal of $S$ if every $l, m, n \in S$ :

1. $\quad l \leq m$ implies $h_{n}(l) \leq h_{n}(m)$ and $h_{p}(l) \geq h_{p}(m)$;

2. $\quad\left(h_{n}(l m n) \leq h_{n}(n)\right.$ with $\left.h_{p}(\operatorname{lmn}) \geq h_{p}(n)\right)\left(\right.$ resp. $\left(h_{n}(\operatorname{lmn}) \leq h_{n}(m)\right.$ with $\left.h_{p}(\operatorname{lmn}) \geq h_{p}(m)\right)$ and $\left(h_{n}(\operatorname{lmn}) \leq h_{n}(l)\right.$ with $\left.h_{p}(\operatorname{lmn}) \geq h_{p}(l)\right)$.

A bipolar fuzzy two-sided ideal can be defined when it is a bipolar fuzzy left and a bipolar fuzzy right ideal. A bipolar fuzzy left, a bipolar fuzzy lateral and a bipolar fuzzy right ideal are collectively called a bipolar fuzzy ideal.

Definition 2. A bipolar fuzzy set $h=\left(S ; h_{n}, h_{p}\right)$ will be called a bipolar fuzzy generalized bi-ideal of $S$ if every $l, m, n, p, q \in S$

1. $\quad l \leq m$ implies $h_{n}(l) \leq h_{n}(m)$ and $h_{p}(l) \geq h_{p}(m)$;

2. $\quad h_{n}($ lpmqn $) \leq \vee\left\{h_{n}(l), h_{n}(m), h_{n}(n)\right\}$ and $h_{p}($ lpmqn $) \geq \wedge\left\{h_{p}(l), h_{p}(m), h_{p}(n)\right\}$.

Definition 3. A bipolar fuzzy set $h=\left(S ; h_{n}, h_{p}\right)$ is called a bipolar fuzzy subsemigroup of $S$ if every $l, m, n, p, q \in S$

1. $\quad l \leq m$ implies $h_{n}(l) \leq h_{n}(m)$ and $h_{p}(l) \geq h_{p}(m)$;

2. $\quad h_{n}(l m n) \leq \vee\left\{h_{n}(l), h_{n}(m), h_{n}(n)\right\}$ and $h_{p}(\operatorname{lmn}) \geq \wedge\left\{h_{p}(l), h_{p}(m), h_{p}(n)\right\}$.

A bipolar fuzzy generalized bi-ideal with the bipolar fuzzy subsemigroup of $S$ is called a bipolar fuzzy bi-ideal of $S$.

Definition 4. If $(s, t) \in[-1,0) \times(0,1], h=\left(S ; h_{n}, h_{p}\right)$ is the bipolar fuzzy set in $S$ where:

$$
h_{n}(m)=\left\{\begin{array}{ll}
s & \text { if } m \in(l], \\
0 & \text { if } m \notin(l],
\end{array} h_{p}(m)= \begin{cases}t & \text { if } m \in(l] \\
0 & \text { if } m \notin(l]\end{cases}\right.
$$

This is called as an ordered bipolar fuzzy point with support $l$ and value $(s, t)$. Symbolically, it is written as $\frac{l}{(s, t)}$. For bipolar fuzzy set $\frac{l}{(s, t)}$ in $S$ and an ordered bipolar fuzzy point $\frac{l}{(s, t)}$, we say that:

1. $\frac{l}{(s, t)} \in h$ if $h_{n}(l) \leq s$ with $h_{p}(l) \geq t$,

2. if $h_{n}(l)+s<-1$ and $h_{p}(l)+t>1$,

3. $\frac{l}{(s, t)} \in \vee q h$ whenever $\frac{l}{(s, t)} \in h$ or $\frac{l}{(s, t)} q h$,

4. $\quad \frac{l}{(s, t)} \in \wedge q h$ whenever $\frac{l}{(s, t)} \in h$ and $\frac{l}{(s, t)} q h$,

5. $\frac{l}{(s, t)} \bar{\alpha} h$ whenever $\frac{l}{(s, t)} \alpha h$ does not hold for $\alpha \in\{\in, q, \in \vee q, \in \wedge q\}$.

Let $h=\left(S ; h_{n}, h_{p}\right)$ be a bipolar fuzzy set in $S$ in such a way that $h_{n}(l) \geq-0.5$ and $h_{p}(l) \leq 0.5$ for every $l \in S$. Suppose that $l \in S$ and $(s, t) \in[-1,0) \times(0,1]$ such that $\frac{l}{(s, t)} \in \wedge q h$. When $\frac{l}{(s, t)} \in h$ and $\frac{l}{(s, t)} \in q h$, we know that $h_{n}(l) \leq s, h_{p}(l) \geq t, h_{n}(l)+s<-1$ and $h_{p}(l)+t>1$. This shows that $-1>h_{n}(l)+s \geq 2 h_{n}(l)$ and $1<h_{p}(l)+t \leq 2 h_{p}(l)$. Thus, $1<2 h_{p}(l)$ implies $0.5<h_{p}(l)$ so that $h_{n}(l)<-0.5$ and $0.5<h_{p}(l)$. This means that $\left\{\frac{l}{(s, t)} \mid \frac{l}{(s, t)} \in \wedge q\right\}=\varphi$. As a result the following case $\alpha=\in \wedge q$ will be omitted.

Definition 5. A bipolar fuzzy set $h=\left(S ; h_{n}, h_{p}\right)$ in $S$ is known as $(\alpha, \beta)$ - bipolar fuzzy generalized bi-ideal of $S$ if every $(s, t) \in[-1,0) \times(0,1]$ and every $l, m, n, p, q \in S$

1. $l \leq m, \frac{m}{(s, t)} \alpha h$ implies $\frac{l}{(s, t)} \beta h$

2. $\frac{l}{\left(s_{1}, t_{1}\right)} \in \alpha h, \frac{m}{\left(s_{2}, t_{2}\right)} \in \alpha h, \frac{n}{\left(s_{3}, t_{3}\right)} \in \alpha h$ implies $\frac{l p m q n}{\left(\vee\left\{s_{1}, s_{2}, s_{3}\right\}, \wedge\left\{t_{1}, t_{2}, t_{3}\right\}\right)} \beta h$. 
Definition 6. Let $u=\left(S ; u_{n}, u_{p}\right)$ be a bipolar fuzzy set in $S$. We define the upper part $u^{+}=\left(S ; u_{n}^{+}, u_{p}^{+}\right)$of $u=\left(S ; u_{n}, u_{p}\right)$ as follows: $u_{n}^{+}(x)=u_{n}(x) \wedge-0.5$ and $u_{p}^{+}(x)=u_{p}(x) \vee 0.5$. In a same way, the lower part $u^{-}=\left(S ; u_{n}^{-}, u_{p}^{-}\right)$of $u=\left(S ; u_{n}, u_{p}\right)$ is defined as: $u_{n}^{-}(x)=u_{n}(x) \wedge-0.5$ and $u_{p}^{-}(x)=u_{p}(x) \vee 0.5$.

Definition 7. Let $F$ be a non-empty subset of $S$. Thus, the upper part $\chi_{F}^{+}=\left(S, \chi_{n F}^{+}, \chi_{p F}^{+}\right)$of the characteristic function $\chi_{F}=\left(S, \chi_{n F}, \chi_{p F}\right)$ of $F$ is explained as $\chi_{n F}^{+}(d)=\left\{\begin{array}{cc}-1 & \text { if } d \in F \\ -0.5 & \text { if } d \notin F\end{array}\right.$ and $\chi_{p F}^{+}(d)=\left\{\begin{array}{cl}1 & \text { if } d \in F \\ 0.5 & \text { if } d \notin F\end{array}\right.$. In the same way, the lower part $\chi_{F}^{-}=\left(S, \chi_{n F}^{-}, \chi_{p F}^{-}\right)$of the characteristic function $\chi_{F}=\left(S, \chi_{n F}, \chi_{p F}\right)$ of $F$ can be represented by $\chi_{n F}^{-}(d)=\left\{\begin{array}{cl}-0.5 & \text { if } d \in F \\ 0 & \text { if } d \notin F\end{array}\right.$ and $\chi_{p F}^{-}(d)=$ $\left\{\begin{array}{cl}0.5 & \text { if } d \in F \\ 0 & \text { if } d \notin F\end{array}\right.$

\section{The Bipolar Fuzzy Sets of an Ordered Ternary Semigroup}

In this section, the concept of the bipolar fuzzy sets of ordered ternary semigroups, bipolar fuzzy left, right and lateral ideals are being discussed with the help of various lemmas and theorems. The following theorem was proved in [21] only for fuzzy sets in an ordered ternary semigroup but here, we have proved it for bipolar fuzzy sets in an ordered ternary semigroup. For bipolar fuzzy sets in ordered semigroups, it has only been defined in literature but not proved. The proof of this theorem is not obvious.

Theorem 1. If $S$ is an ordered ternary groupoid, $(B F(S), 0, \leq)$ is also an ordered ternary groupoid. In addition, if $S$ is an ordered ternary semigroup, $(B F(S), 0, \leq)$ is also an ordered ternary semigroup.

Proof. To prove that $(B F(S), \circ, \leq)$ is an ordered ternary groupoid, it is enough to show that $h_{1 p} \circ$ $h_{2 p} \circ h_{3 p} \leq g_{1 p} \circ g_{2 p} \circ g_{3 p}$ and $h_{1 n} \circ h_{2 n} \circ h_{3 n} \geq g_{1 n} \circ g_{2 n} \circ g_{3 n}$ for all $h_{i}$, as well as $g_{i} \in B F(S)$ with $h_{i} \leq g_{i}$ where $i=1,2,3$. Indeed, as we know that $a \in S$ if $A_{a} \neq \varphi$, we have $\left(h_{1 p} \circ h_{2 p} \circ\right.$ $\left.h_{3 p}\right)(a)=\underset{(l, m, n) \in A_{a}}{\vee} \min \left\{h_{1_{p}}(l), h_{2_{p}}(m), h_{3_{p}}(n)\right\} \leq \underset{(l, m, n) \in A_{a}}{\vee} \min \left\{g_{1_{p}}(l), g_{2_{p}}(m), g_{3_{p}}(n)\right\} \leq\left(g_{1 p} \circ\right.$ $\left.g_{2_{p}} \circ g_{3_{p}}\right)(a)$. Furthermore, $\left(h_{1 n} \circ h_{2 n} \circ h_{3 n}\right)(a)=\bigwedge_{(l, m, n) \in A_{a}}^{\wedge} \max \left\{h_{1 n}(l), h_{2 n}(m), h_{3 n}(n)\right\} \geq \hat{\wedge} \wedge A_{a}$ $\max \left\{g_{1 n}(l), g_{2 n}(m), g_{3 n}(n)\right\} \geq\left(g_{1 n} \circ g_{2 n} \circ g_{3 n}\right)(a)$. Now if we assume that $S$ is an ordered ternary semigroup, we have to check only the associative law: $h_{1} \circ h_{2} \circ\left(h_{3} \circ h_{4} \circ h_{5}\right)=h_{1} \circ\left(h_{2} \circ h_{3} \circ h_{4}\right) \circ h_{5}=$ $\left(h_{1} \circ h_{2} \circ h_{3}\right) \circ h_{4} \circ h_{5}$ for all $h_{1}, h_{2}, h_{3}, h_{4}, h_{5} \in B F(S)$. Only the first equality is justified here. Let $a \in S$ with $A_{a} \neq \varphi$.

$$
\begin{aligned}
{\left[h_{1_{p}} \circ\left(h_{2_{p}} \circ h_{3_{p}} \circ h_{4_{p}}\right) \circ h_{5_{p}}\right](a) } & =\underset{\left(p^{\prime}, \mu, n\right) \in A_{a}}{\vee}\left\{h_{1_{p}}\left(p^{\prime}\right) \wedge\left(h_{2_{p}} \circ h_{3_{p}} \circ h_{4_{p}}\right)(\mu) \wedge h_{5_{p}}(n)\right\} . \\
{\left[\left(h_{1_{p}} \circ h_{2_{p}} \circ h_{3_{p}}\right) \circ h_{4_{p}} \circ h_{5_{p}}\right](a) } & =\underset{(l, m, n) \in A_{a}}{\vee}\left\{\left(h_{1_{p}} \circ h_{2_{p}} \circ h_{3_{p}}\right)(l) \wedge h_{4_{p}}(m) \wedge h_{5_{p}}(n)\right\} \\
& =\underset{\left(p^{\prime \prime}, q^{\prime}, r^{\prime}, m, n\right) \in A_{1}}{\vee}\left\{\begin{array}{c}
\left(\left(h_{1_{p}}\left(p^{\prime \prime}\right) \wedge h_{2_{p}}\left(q^{\prime}\right) \wedge h_{3_{p}}\right)\left(r^{\prime}\right)\right) \wedge \\
h_{4_{p}}(m) \wedge h_{5_{p}}(n)
\end{array}\right\}
\end{aligned}
$$

Without a loss of generality, we suppose that $A_{l} \neq \varphi$ for some $(l, m, n) \in A_{a}$ and $A_{\mu} \neq \varphi$ for some $\left(p^{\prime}, \mu, n\right) \in A_{a}$. This is because $A_{l}=\varphi$ for any $(l, m, n) \in A_{a}$ if and only if $A_{u}=\varphi$ for some $\left(p^{\prime}, \mu, n\right) \in A_{a}$. In contrast, $A_{1}=\left\{\left(p^{\prime \prime}, q^{\prime}, r^{\prime}, m, n\right) \mid\left(p^{\prime \prime}, q^{\prime}, r^{\prime}\right) \in A_{l}\right.$ and $(l, m, n) \in A_{a}$ for any $\left.l \in S\right\}$. In the same way, 


$$
\left[h_{1 p} \circ\left(h_{2 p} \circ h_{3 p} \circ h_{4 p}\right) \circ h_{5 p}\right](a)=\underset{\left(p^{\prime \prime}, q^{\prime}, r^{\prime}, m, n\right) \in A_{2}}{\vee}\left\{h_{1 p}\left(p^{\prime \prime}\right) \wedge\left(h_{2 p}\left(q^{\prime}\right) \wedge\left(h_{3 p}\right)\left(r^{\prime}\right) \wedge h_{4 p}(m)\right) \wedge h_{5 p}(n)\right\}
$$

where $A_{2}=\left\{\left(p^{\prime \prime}, q^{\prime}, r^{\prime}, m, n\right) \mid\left(q^{\prime}, r^{\prime}, m\right) \in A_{\mu}\right.$ and $(l, m, n) \in A_{a}$ for any $\left.u \in S\right\}$. It is sufficient to show that $A_{1}=A_{2}$. Let $\left(p^{\prime \prime}, q^{\prime}, r^{\prime}, m, n\right) \in A_{1}$ and thus for any $l \in S$ we have $a \leq l m n$ and $l \leq p^{\prime \prime} q^{\prime} r^{\prime}$. If we suppose $\mu=q^{\prime} r^{\prime} m$ we have $a \leq l m n \leq p^{\prime \prime} q^{\prime} r^{\prime} m n \leq p^{\prime \prime} \mu n$ and $\mu \leq q^{\prime} r^{\prime} m$. This shows that $\left(p^{\prime \prime}, \mu, n\right) \in A_{a}$ and $\left(q^{\prime}, r^{\prime}, m\right) \in A_{\mu}$. Therefore, $\left(p^{\prime \prime}, q^{\prime}, r^{\prime}, m, n\right) \in A_{2}$ and subsequently $A_{1} \subseteq A_{2}$. Similarly, $A_{2} \subseteq A_{1}$ and therefore, $A_{1}=A_{2}$ is required. Hence we can prove the negative part by using similar arguments.

A binary operation $\circ$ over $B F(S)$ is defined by $*$ to avoid from any confusion for any $a \in S$, where $S$ is an ordered semigroup. Let $B_{a}=\{(l, m) \in S \times S \mid a \leq l m\}$. For any two bipolar fuzzy sets $h=\left(S ; h_{n}, h_{p}\right)$ and $g=\left(S ; g_{n}, g_{p}\right)$ of $S, h * g$ is defined as $h * g=\left(S ; h_{n} \circ g_{n}, h_{p} \circ g_{p}\right)$

$$
\begin{aligned}
& \left(h_{n} * g_{n}\right)(a)=\left\{\begin{array}{cc}
\left\{h_{n}(l) \vee g_{n}(m)\right\} & \text { if } B_{a} \neq \varphi \\
0 & \text { if } B_{a}=\varphi
\end{array},\right. \\
& \left(h_{p} * g_{p}\right)(a)=\left\{\begin{array}{cc}
\left\{h_{p}(l) \wedge g_{p}(m)\right\} & \text { if } B_{a} \neq \varphi \\
0 & \text { if } B_{a}=\varphi .
\end{array}\right.
\end{aligned}
$$

Theorem 2. Suppose that $S$ is an ordered semigroup. If $S$ is regarded as an ordered ternary semigroup through a usual natural method, the ternary operation of $B F(S)$ is persuaded through the binary operation $*$ of $B F(S)$.

Proof. If we let $a \in S$ with $A_{a}=\varphi$, we obtain $\left(h_{p} \circ g_{p} \circ f_{p}\right)(a)=0$. When $B_{a}=\varphi$, we obtain $\left(h_{p} * g_{p} * f_{p}\right)(a)=0$. If $B_{a} \neq \varphi$, this reveals that $B_{m}=\varphi$ for all $(m, n) \in B_{a}$ and so, $\left(h_{p} * g_{p} * f_{p}\right)(a)=$ $\underset{(m, n) \in B_{a}}{\vee}\left\{\left(h_{p} * g_{p}\right)(m) \wedge f_{p}(n)\right\}=0$. Thus, $\left(h_{p} * g_{p} * f_{p}\right)(a)=\left(h_{p} \circ g_{p} \circ f_{p}\right)(a)$. Suppose $a \in S$ such that $A_{a} \neq \varphi$. Thus, $B_{a} \neq \varphi$ and $B_{m} \neq \varphi$ for some $(m, n) \in B_{a}$. Thus, $\left(h_{p} * g_{p} * f_{p}\right)(a)=$ $\underset{(m, n) \in B_{a}}{\vee}\left\{\left(h_{p} * g_{p}\right)(m) \wedge f_{p}(n)\right\}$. This becomes $\left(h_{p} * g_{p} * f_{p}\right)(a)=\underset{(k, l, n) \in A}{\vee}\left\{h_{p}(k) \wedge g_{p}(l) \wedge f_{p}(n)\right\}$. Here, $A=\left\{(k, l, n) \mid(k, l) \in B_{m}\right.$ with $(m, n) \in B_{a}$ for any $\left.m \in S\right\}$. To prove $A \subseteq A_{a}$, let $(k, l, n) \in A$ such that $(k, l) \in B_{m}$ and $(m, n) \in B_{a}$. This implies $a \leq m n$ and $m \leq k l$. Thus, $a \leq m n \leq k \ln$ and $(k, l, n) \in A_{a}$. Thus, $A \subseteq A_{a}$. On the contrary, let $(k, l, n) \in A_{a}$ with $m=k l$. Thus, $a \leq k \ln \leq m n$ and $m \leq k l$. Thus, $(k, l) \in B_{m}$ and $(m, n) \in B_{a}$. Consequently, $(k, l, n) \in A$. This implies that $A_{a} \subseteq A$. Therefore, $A=A_{a}$ so $\left(h_{p} * g_{p} * f_{p}\right)(a)=\left(h_{p} \circ g_{p} \circ f_{p}\right)(a)$. Similarly, we can prove that $\left(h_{n} * g_{n} * f_{n}\right)=\left(h_{n} \circ g_{n} \circ f_{n}\right)$.

We now remark that if $\left(S^{3}\right]=S$, the left ideals, right ideals and ideals of the ordered semigroup $S$ are the same as the left ideals, right ideals and two-sided ideals of the ordered ternary semigroup $S$, respectively. The same conclusions hold for bipolar fuzzy ideals.

\section{Bipolar Fuzzy Generalized Bi-Ideals}

In this paper, $S$ means an ordered ternary semigroup unless otherwise mentioned. Each bipolar fuzzy bi-ideal is also a bipolar fuzzy generalized bi-ideal of $S$ but the reverse inclusion is not valid. For the justification of this remark, we provide the following example.

Example 2. Let $S$ be an ordered ternary semigroup as defined in example 1, which also defines a bipolar fuzzy set $h=\left(S ; h_{n}, h_{p}\right)$ of an ordered ternary semigroup $S$. Thus, every bipolar fuzzy set $h=\left(S ; h_{n}, h_{p}\right)$ of an ordered ternary semigroup that satisfies conditions 1 and 2 of Definition 5 are the bipolar fuzzy generalized bi-ideals of an ordered ternary semigroup. Bipolar fuzzy generalized bi-ideals are not bipolar fuzzy bi-ideals. The bipolar fuzzy set defined as follows 


$\begin{array}{rrrrr}S & \alpha & \beta & \gamma & \delta \\ h_{n} & -0.7 & -0.1 & -0.5 & -0.2 \\ h_{p} & 0.8 & 0.2 & 0.4 & 0.3\end{array}$

is a bipolar fuzzy generalized bi-ideal of $S$ but this is not bipolar fuzzy bi-ideal of $S$, because $-0.1=h_{n}(\beta)=$ $h_{n}(\gamma \cdot \gamma \cdot \gamma)>\vee\left\{h_{n}(\gamma), h_{n}(\gamma), h_{n}(\gamma)\right\}=\vee\{-0.5,-0.5,-0.5\}=-0.5$ and $0.2=h_{p}(\beta)=h_{p}(\gamma \cdot \gamma \cdot \gamma)<$ $\wedge\left\{h_{p}(\gamma), h_{p}(\gamma), h_{p}(\gamma)\right\}=\wedge\{0.4,0.4,0.4\}=0.4$. This example justifies the above remark.

Lemma 1. [23] Considering $S$ as an ordered ternary semigroup, the below given properties are equivalent:

1. $S$ is regular.

2. $\quad F \cap I=(F I F]$ for each generalized bi-ideal $F$ and every ideal $I$ of $S$.

Lemma 2. [24] Considering $S$ as an ordered ternary semigroup, the below given properties are equivalent:

1. $S$ is regular.

2. $F \cap L \subseteq(F S L]$ for every bi-ideal $F$ and every left ideal $L$ of $S$.

3. $R \cap F \cap L \subseteq(R S F S L]$ for every bi-ideal $F$, every right ideal $R$ and every left ideal $L$ of $S$.

For a bipolar fuzzy set $h=\left(S ; h_{n}, h_{p}\right)$ of $S$ with $(s, t) \in[-1,0) \times(0,1]$, we define $N(h ; s)=$ $\left\{l \in S \mid h_{n}(l) \leq s\right\}$ and $P(h ; t)=\left\{l \in S \mid h_{p}(l) \geq t\right\}$, which are known as the negative $s$-cut and positive $t$-cut of $h=\left(S ; h_{n}, h_{p}\right)$, respectively. $(s, t)$-cut of $h=\left(S ; h_{n}, h_{p}\right)$ is denoted by $C(h ;(s, t))$ and is defined as $C(h ;(s, t))=N(h ; s) \cap P(h ; t)[20]$

Here, we characterize bipolar fuzzy generalized bi-ideal of $S$ in the form of $(s, t)$-cut generalized bi-ideal of $S$.

Theorem 3. Let $h=\left(S ; h_{n}, h_{p}\right)$ be a bipolar fuzzy set in $S$. Thus, $h=\left(S ; h_{n}, h_{p}\right)$ is a bipolar fuzzy generalized bi-ideal of an ordered ternary semigroup $S$ if and only if the non-empty $(s, t)$-cut $C(h ;(s, t))$ belonging to $h=\left(S ; h_{n}, h_{p}\right)$ is termed as a generalized bi-ideal of $S$ for every $(s, t) \in[-1,0) \times(0,1]$.

If $F$ is a subset of $S$, the characteristic function of $F$ is denoted by $\chi_{F}^{-}=\left(S ; \chi_{n F}^{-}, \chi_{p F}^{-}\right)$where $\chi_{n F}^{-}$ and $\chi_{p F}^{-}$are described as $\chi_{n F}^{-}(d)=\left\{\begin{array}{cc}-1 & \text { if } d \in F \\ 0 & \text { if } d \notin F\end{array}\right.$ and $\chi_{p F}^{-}(d)=\left\{\begin{array}{cc}1 & \text { if } d \in F \\ 0 & \text { if } d \notin F\end{array}\right.$.

\section{5. $(\alpha, \beta)$-Bipolar Fuzzy Generalized Bi-Ideals}

The $(\in, \in \vee q)$-bipolar fuzzy generalized bi-ideal has played a central role in the $(\alpha, \beta)$-bipolar fuzzy generalized bi-ideal. The characterizations of the regular ordered semigroups in terms of $(\alpha, \beta)$-fuzzy generalized bi-ideals and in terms of $(\alpha, \beta)$-bipolar fuzzy generalized bi-ideals were given in $[3,20]$. Here, we extend their generalizations to ordered ternary semigroups.

Theorem 4. Let $h=\left(S ; h_{n}, h_{p}\right)$ be a bipolar fuzzy set in $S$. Thus, $h=\left(S ; h_{n}, h_{p}\right)$ is a bipolar fuzzy generalized bi-ideal of $S$ if and only if every $(s, t) \in[-1,0) \times(0,1]$ and every $l, m, n, p, q \in S$

$1 \quad l \leq m, \frac{m}{(s, t)} \in h$ implies $\frac{l}{(s, t)} \in h$

$2 \frac{l}{\left(s_{1}, t_{1}\right)} \in h, \frac{m}{\left(s_{2}, t_{2}\right)} \in h$ and $\frac{n}{\left(s_{3}, t_{3}\right)} \in h$ implies that $\frac{l p m q n}{\left(\vee\left\{s_{1}, s_{2}, s_{3}\right\}, \wedge\left\{t_{1}, t_{2}, t_{3}\right\}\right)} \in h$.

Proof. Let $h=\left(S ; h_{n}, h_{p}\right)$ be a bipolar fuzzy generalized bi-ideal of $S$. Let $l, m \in S$ such that $l \leq m$ and $\frac{m}{(s, t)} \in h$. Thus, $h_{n}(m) \leq s$ and $h_{p}(m) \geq t$. As $h=\left(S ; h_{n}, h_{p}\right)$ is a bipolar fuzzy generalized bi-ideal of $S$, we get $h_{n}(l) \leq h_{n}(m)$ and $h_{p}(l) \geq h_{p}(m)$ for $l \leq m$. This shows that $h_{n}(l) \leq s$ and $h_{p}(l) \geq t$ so $\frac{l}{(s, t)} \in h$. Consider $l, m, n, p, q \in S$ and $\left(s_{1}, t_{1}\right),\left(s_{2}, t_{2}\right),\left(s_{3}, t_{3}\right) \in[-1,0) \times(0,1]$ such that $\frac{l}{\left(s_{1}, t_{1}\right)} \in h$, $\frac{m}{\left(s_{2}, t_{2}\right)} \in h$ and $\frac{n}{\left(s_{3}, t_{3}\right)} \in h$. Thus, $h_{n}(l) \leq s_{1}, h_{n}(m) \leq s_{2}, h_{n}(n) \leq s_{3}$ and $h_{p}(l) \geq t_{1}, h_{p}(m) \geq t_{2}$, 
$h_{p}(n) \geq t_{3}$. This follows Definition 10 as $h_{n}(l p m q n) \leq \vee\left\{h_{n}(l), h_{n}(m), h_{n}(n)\right\} \leq \vee\left\{s_{1}, s_{2}, s_{3}\right\}$ and $h_{p}($ lpmqn $) \geq \wedge\left\{h_{p}(l), h_{p}(m), h_{p}(n)\right\} \geq \wedge\left\{t_{1}, t_{2}, t_{3}\right\}$. Thus, $\frac{l p m q n}{\left(\vee\left\{s_{1}, s_{2}, s_{3}\right\}, \wedge\left\{t_{1}, t_{2}, t_{3}\right\}\right)} \in h$.

Conversely consider $l, m \in S$ such that $l \leq m$. Let $h_{n}(m)=s$ and $h_{p}(l)=t$. Thus, $\frac{m}{(s, t)} \in h$ implies that $\frac{l}{(s, t)} \in h$. Thus, $h_{n}(l) \leq s$ and $h_{p}(l) \geq t$. Therefore, $h_{n}(l) \leq s=h_{n}(m)$ and $h_{p}(l) \geq t=h_{p}(m)$. Let $l, m, n, p, q \in S$ since $\frac{l}{\left(h_{n}(l), h_{p}(l)\right)} \in h, \frac{m}{\left(h_{n}(m), h_{p}(m)\right.} \in h$ and $\frac{n}{\left(h_{n}(n), h_{p}(n)\right)} \in h$ for every $l, m, n \in S$. Thus, by (2), we have $\frac{l p m q n}{\left(\vee\left\{h_{n}(l), h_{n}(m), h_{n}(n)\right\}, \wedge\left\{h_{p}(l), h_{p}(m), h_{p}(n)\right\}\right)} \in h$. This implies that $h_{n}(l p m q n) \leq \vee\left\{h_{n}(l), h_{n}(m), h_{n}(n)\right\}$ and $h_{p}(l p m q n) \geq \wedge\left\{h_{p}(l), h_{p}(m), h_{p}(n)\right\}$. This theorem is proved using Definition 5.

Theorem 5. Let $h=\left(S ; h_{n}, h_{p}\right)$ be a non-zero $(\alpha, \beta)$-bipolar fuzzy generalized bi-ideal of $S$. Thus, we can determine that the set $S_{\circ}=\left\{l \in S \mid h_{n}(l) \neq 0\right\} \cap\left\{l \in S \mid h_{p}(l) \neq 0\right\}$ is a generalized bi-ideal of $S$.

Proof. Let $l, m \in S, l \leq m$ with $m \in S_{\circ}$. At that moment, $h_{n}(m) \neq 0 \neq h_{p}(m)$. Suppose $h_{n}(l)=0=h_{p}(l)$. When $\alpha \in\{\in, \in \vee q\}$, we can determine that $\frac{m}{\left(h_{n}(m), h_{p}(m)\right)} \alpha h$ but $\frac{l}{\left(h_{n}(m), h_{p}(m)\right)} \bar{\beta} h$ for every $\beta \in\{\in, q, \in \vee q, \in \wedge q\}$. This contradicts the supposed statement. Thus, $h_{n}(l) \neq 0 \neq h_{p}(l)$, $h_{n}(m) \neq 0 \neq h_{p}(m)$ and $h_{n}(n) \neq 0 \neq h_{p}(n)$. Assume that $h_{n}\left(\right.$ lpmqn) $=0=h_{n}($ lpmqn). If $\alpha \in\{\in, \in \vee q\}$, we can determine that $\frac{l}{\left(h_{n}(l), h_{p}(l)\right)} \alpha h, \frac{m}{\left(h_{n}(m), h_{p}(m)\right)} \alpha h$ and $\frac{n}{\left(h_{n}(n), h_{p}(n)\right)} \alpha h$ but $\frac{l p m q n}{\left(\left(h_{n}(l) \vee h_{n}(m) \vee h_{n}(n)\right),\left(h_{p}(l) \wedge h_{p}(m) \wedge h_{p}(n)\right)\right.} \bar{\beta} h$ for all $\beta \in\{\in, q, \in \vee q, \in \wedge q\}$. This shows a contradiction. Thus, $h_{n}(l p m q n) \neq 0 \neq h_{p}(l p m q n)$. This shows that $l p m q n \in S_{\circ}$.

\section{6. $(\in, \in \vee q)$-Bipolar Fuzzy Generalized Bi-Ideals}

This section is our most important section as our main contribution is in this section. We have proved that if a bipolar fuzzy set $h=\left(S ; h_{n}, h_{p}\right)$ in the ordered ternary semigroup $S$ is the $(\in, \in$ $\vee q$ )-bipolar fuzzy generalized bi-ideal $S$, we know that it satisfies two particular conditions. We have proved in an example that the converse of this result is not true in general. However, this result was previously proved in [20] for the ordered semigroup with if and only if conditions. However, we negate this by giving an example, which proves that the converse is not true.

Theorem 6. If $F$ is the left (resp. right, lateral) ideal of $S$ we can determine that $\chi_{F}=\left(S ; \chi_{n F}, \chi_{p F}\right)$ is the bipolar fuzzy left (resp. right, lateral) ideal of $S$.

Proof. Let $l, m, n \in S$. Case (1) occurs when $l, m, n \notin F$, which means that $\chi_{n F}(l)=0=\chi_{n F}(m)=$ $\chi_{n F}(n)$ and $\chi_{p F}(l)=0=\chi_{p F}(m)=\chi_{p F}(n)$. This implies that $\chi_{n F}(\operatorname{lm} n) \leq 0=\chi_{n F}(n)$ and $\chi_{p F}(\operatorname{lmn}) \geq$ $0=\chi_{p F}(n)$. Case (2) occurs if $l, m, n \in F$. Thus, $\chi_{n F}(l)=-1=\chi_{n F}(m)=\chi_{n F}(n)$ and $\chi_{p F}(l)=1=$ $\chi_{p F}(m)=\chi_{p F}(n)$ so $\chi_{n F}(l m n) \leq-1=\chi_{n F}(n)$ and $\chi_{p F}(l m n) \leq 1=\chi_{p F}(n)$. Case (3) occurs if $l \in F$ but $m, n \notin F$, which means that $\chi_{n F}(l)=-1$ but $\chi_{n F}(m)=0=\chi_{n F}(n)$. Furthermore, $\chi_{p F}(l)=1$ but $\chi_{p F}(m)=0=\chi_{p F}(n)$. Thus, $\chi_{n F}(\operatorname{lm} n) \leq 0=\chi_{n F}(n)$ and $\chi_{p F}(\operatorname{lm} n) \geq 0=\chi_{n F}(n)$ as $\operatorname{lm} n \in F$ or $\operatorname{lm} n \notin F$. Similarly, these relations hold for other cases. Hence, $\chi_{F}$ is a bipolar fuzzy left ideal of $S$.

Theorem 7. If $F$ is generalized bi-ideal of $S$, we can determine that $\chi_{F}=\left(S ; \chi_{n F}, \chi_{p F}\right)$ is a bipolar fuzzy generalized bi-ideal of $S$.

Proof. We have to prove the following inequalities for all $l, m, n, p, q \in S$ :

$\chi_{n F}(l p m q n) \leq \max \left\{\chi_{n F}(l), \chi_{n F}(m), \chi_{n F}(n)\right\}$ and $\chi_{p F}(l p m q n) \geq \min \left\{\chi_{p F}(l), \chi_{p F}(m), \chi_{p F}(n)\right\}$. Let $l, m, n, p, q \in S$. Case (1) occurs when $l, m, n, p, q \notin F$, which means that $\chi_{n F}(l)=0=$ $\chi_{n F}(m)=\chi_{n F}(n)=\chi_{n F}(p)=\chi_{n F}(q)$ and $\chi_{p F}(l)=0=\chi_{p F}(m)=\chi_{p F}(n)=\chi_{p F}(p)=\chi_{p F}(q)$. This implies that $\chi_{n F}($ lpmqn $) \leq 0=\max \left\{\chi_{n F}(l), \chi_{n F}(m), \chi_{n F}(n)\right\}$ and $\chi_{p F}($ lpmqn) $\geq 0=$ $\min \left\{\chi_{p F}(l), \chi_{p F}(m), \chi_{p F}(n)\right\}$. Case (2) occurs when $l, m, n, p, q \in F$, which means that $\chi_{n F}(l)=$ $-1=\chi_{n F}(m)=\chi_{n F}(n)=\chi_{n F}(p)=\chi_{n F}(q)$ and $\chi_{p F}(l)=\chi_{p F}(m)=\chi_{p F}(n)=\chi_{p F}(p)=1=\chi_{p F}(q)$. 
Since $F$ is a generalized bi-ideal of $S$ we have $\chi_{n F}(l p m q n)=-1=\max \left\{\chi_{n F}(l), \chi_{n F}(m), \chi_{n F}(n)\right\}$ and $\chi_{p F}(l p m q n)=1=\min \left\{\chi_{p F}(l), \chi_{p F}(m), \chi_{p F}(n)\right\}$. For Case (3), if $l \in F$ but $m, n, p, q \notin F$, we determine that $\chi_{n F}(l)=-1,0=\chi_{n F}(m)=\chi_{n F}(n)=\chi_{n F}(p)=\chi_{n F}(q), \chi_{p F}(l)=1$ and $0=\chi_{n F}(m)=\chi_{n F}(n)=$ $\chi_{n F}(p)=\chi_{n F}(q)$. This implies that $\chi_{n F}(l p m q n)=-1 \leq 0=\max \left\{\chi_{n F}(l), \chi_{n F}(m), \chi_{n F}(n)\right\}$ and $\chi_{p F}(l p m q n) \geq 0=\min \left\{\chi_{p F}(l), \chi_{p F}(m), \chi_{p F}(n)\right\}$. Similarly, these relations hold for other cases. Hence, $\chi_{F}$ is a bipolar fuzzy generalized bi-ideal of $S$.

Theorem 8. If a bipolar fuzzy set $h=\left(S ; h_{n}, h_{p}\right)$ in $S$ is a $(\in, \in \vee q)$-bipolar fuzzy generalized bi-ideal $S$, we can determine that the following properties are being followed. For every $l, m, n, p, q \in S$ :

1. $l \leq m$ implies $h_{n}(l) \leq \vee\left\{h_{n}(m),-0.5\right\}$ and $h_{p}(l) \geq \wedge\left\{h_{p}(m),-0.5\right\}$

2. $\quad h_{n}(l p m q n) \leq \vee\left\{h_{n}(l), h_{n}(m), h_{n}(n),-0.5\right\}$ and $h_{p}(l p m q n) \geq \wedge\left\{h_{p}(l), h_{p}(m), h_{p}(n), 0.5\right\}$.

Proof. Let $h=\left(S ; h_{n}, h_{p}\right)$ be an $(\in, \in \vee q)$-bipolar fuzzy generalized bi-ideal $S$. Suppose $l, m \in S$ with $l \leq m$. Considering the following cases:

(1) $h_{n}(m)>-0.5$ and $h_{p}(m)<0.5$,

(2) $h_{n}(m) \leq 0.5$ and $h_{p}(m) \geq 0.5$,

(3) $h_{n}(m) \leq-0.5$ and $h_{p}(m)<0.5$,

(4) $h_{n}(m)>-0.5$ and $h_{p}(m) \geq 0.5$,

From case (1), we assume with a contradiction that $h_{n}(l)>\vee\left\{h_{n}(m),-0.5\right\}$ or $h_{p}(l)<$ $\wedge\left\{h_{p}(m), 0.5\right\}$. This makes $h_{n}(l)>h_{n}(m)$ or $h_{p}(l)<h_{p}(m)$. If $h_{n}(l)>h_{n}(m)$, we can determine that there exists $s \in[-1,0)$ such that $h_{n}(l)>s \geq h_{n}(m)$. If we let $t=h_{p}(m)$, we can determine that $\frac{m}{(s, t)} \in h$ but we know that $\frac{l}{(s, t)} \bar{\in}$ and $\frac{l}{(s, t)} \bar{q} h$. That is, we obtain $\frac{l}{(s, t)} \overline{\in V q} h$, which is a contradiction. If $h_{p}(l)<h_{p}(m)$, there exists $t \in(0,1]$ such that $h_{p}(l)<t \leq h_{p}(m)$. If we let $s=h_{n}(m)$, we can determine that $\frac{m}{(s, t)} \in h$ but we know that $\frac{m}{(s, t)} \bar{\in} h$ and $\frac{m}{(s, t)} \bar{q} h$. That is, we obtain $\frac{l}{(s, t)} \overline{\in V q} h$, which is a contradiction. Hence, $h_{n}(l) \leq \vee\left\{h_{n}(m),-0.5\right\}$ or $h_{p}(l) \geq \wedge\left\{h_{p}(m), 0.5\right\}$.

From case (2), we have $h_{n}(m) \leq-0.5$ and $h_{p}(m) \geq 0.5$. This implies $\frac{m}{(-0.5,0.5)} \in h$. Since $\frac{m}{(-0.5,0.5)} \in h$, we can determine that $\frac{l}{(-0.5,0.5)} \in h$. Hence $\frac{l}{(-0.5,0.5)} \in \vee q h$. Thus $\frac{l}{(-0.5,0.5)} \in h$ or $\frac{l}{(-0.5,0.5)} q h$. If $\frac{l}{(-0.5,0.5)} \in h$ we can determine that $h_{n}(l) \leq-0.5 \leq \vee\left\{h_{n}(m),-0.5\right\}$ and $h_{p}(l) \geq 0.5 \geq$ $\wedge\left\{h_{p}(m), 0.5\right\}$. If $\frac{l}{(-0.5,0.5)} q h$, we can determine that $h_{n}(l)-0.5<-1$ and $h_{p}(l)+0.5>1$. This implies that $h_{n}(l)<-0.5$ and $h_{p}(l)>0.5$. Therefore, $h_{n}(l) \leq \vee\left\{h_{n}(m),-0.5\right\}$ and $h_{p}(l) \geq \wedge\left\{h_{p}(m), 0.5\right\}$.

From case (3), we have $h_{n}(m) \leq-0.5$ and $h_{p}(m)<0.5$. Suppose that $h_{n}(l)>-0.5$ or $h_{p}(l)<$ $h_{p}(m)$. If $h_{n}(l)>-0.5$ we can determine that there exists $s \in[-1,0)$ such that $h_{n}(l)>s \geq-0.5 \geq$ $h_{n}(m)$ (using case 3 ). Let $h_{p}(m)=t$ and $h_{n}(m) \leq s$. This represents $\frac{m}{(s, t)} \in h$ on the other side $\frac{l}{(s, t)} \bar{\in} h$ and $\frac{l}{(s, t)} \bar{q} h$. That is, we obtain $\frac{l}{(s, t)} \overline{\in V q}$, which is a contradiction. Now if $h_{p}(l)<h_{p}(m)$ this shows that there exists $t \in(0,1]$ such that $h_{p}(l)<t \leq h_{p}(m)$. If we let $h_{n}(m)=s$, we can determine that $\frac{m}{(s, t)} \in h$ but we know that $\frac{l}{(s, t)} \bar{\in} h$ and $\frac{l}{(s, t)} \bar{q} h$. This implies $\frac{l}{(s, t)} \overline{\in \vee q}$, which is a contradiction. Hence, $h_{n}(l) \leq-0.5 \leq \vee\left\{h_{n}(m),-0.5\right\}$ and $h_{p}(l) \geq h_{p}(m) \geq \wedge\left\{h_{p}(m), 0.5\right\}$.

From case (4). we have $h_{n}(l) \leq h_{n}(m)$ and $h_{p}(l) \geq h_{p}(m) \geq 0.5$. We suppose $h_{n}(l)>h_{n}(m)$ or $h_{p}(l)<0.5$ in a contradiction. If $h_{n}(l)>h_{n}(m)$, we can determine that there exists $s \in[-1,0)$ such that $h_{n}(l)>s \geq h_{n}(m)$. Since $h_{n}(m) \leq s$ and $h_{p}(m) \geq 0.5$ implies $\frac{m}{(s, 0.5)} \in h$, it is impossible to determine $\frac{l}{(s, 0.5)} \bar{\in} h$ and $\frac{l}{(s, 0.5)} \bar{q} h$ that has $\frac{l}{(s, 0.5)} \overline{\in \vee q} h$. If $h_{p}(l)<0.5$, we can determine that there exists $t \in(0,1]$ such that $h_{p}(l)<t<0.5 \leq h_{p}(m)$. Let $s=h_{n}(m)$ and $t \leq h_{p}(m)$. This implies $\frac{m}{(s, t)} \in h$ but we know that $\frac{l}{(s, t)} \bar{\in} h$ and $\frac{l}{(x, t)} \bar{q} h$. That is, we obtain $\frac{l}{(s, t)} \overline{\in V q} h$, which is a contradiction. Hence, $h_{n}(l) \leq h_{n}(m) \leq \vee\left\{h_{n}(m),-0.5\right\}$ and $h_{p}(l) \geq 0.5 \geq \wedge\left\{h_{p}(m), 0.5\right\}$. Thus, the required result is proved. Let $p, q, l, m, n \in S$ and consider the following cases. 
(1) $\vee\left\{h_{n}(l), h_{n}(m), h_{n}(n)\right\}>-0.5$ and $\wedge\left\{h_{p}(l), h_{p}(m), h_{p}(n)\right\}<0.5$,

(2) $\vee\left\{h_{n}(l), h_{n}(m), h_{n}(n)\right\} \leq-0.5$ and $\wedge\left\{h_{p}(l), h_{p}(m), h_{p}(n)\right\} \geq 0.5$,

(3) $\vee\left\{h_{n}(l), h_{n}(m), h_{n}(n)\right\} \leq-0.5$ and $\wedge\left\{h_{p}(l), h_{p}(m), h_{p}(n)\right\}<0.5$,

(4) $\vee\left\{h_{n}(l), h_{n}(m), h_{n}(n)\right\} \geq-0.5$ and $\wedge\left\{h_{p}(l), h_{p}(m), h_{p}(n)\right\} \geq 0.5$

Let $h_{n}(l p m q n)>\vee\left\{h_{n}(l), h_{n}(m), h_{n}(n),-0.5\right\}$ or $h_{p}(l p m q n)<\wedge\left\{h_{p}(l), h_{p}(m), h_{p}(n), 0.5\right\}$.

For the case (1) equation, it becomes $h_{n}($ lpmqn $)>\vee\left\{h_{n}(l), h_{n}(m), h_{n}(n)\right\}$ or $h_{p}($ lpmqn $)<$ $\wedge\left\{h_{p}(l), h_{p}(m), h_{p}(n)\right\}$. If $h_{n}(l p m q n)>\vee\left\{h_{n}(l), h_{n}(m), h_{n}(n)\right\}$, we can determine that there exists $s \in[-1,0)$ such that $h_{n}($ lpmqn $)>s \geq \vee\left\{h_{n}(l), h_{n}(m), h_{n}(n)\right\}$. Let $t=\wedge\left\{h_{p}(l), h_{p}(m), h_{p}(n)\right\}$. Now $\frac{l}{(s, t)} \in h, \frac{m}{(s, t)} \in h$ and $\frac{n}{(s, t)} \in h$ but we know that $\frac{l p m q n}{(s, t)} \bar{\in}$ and $\frac{l p m q n}{(s, t)} \bar{q} h$. This implies $\frac{l p m q n}{(s, t)} \overline{\in V q}$, which is a contradiction. If $h_{p}($ lpmqn $)<\wedge\left\{h_{p}(l), h_{p}(m), h_{p}(n)\right\}$, we can determine that there occurs $t \in(0,1]$ such that $h_{p}($ lpmqn $)<t \leq \wedge\left\{h_{p}(l), h_{p}(m), h_{p}(n)\right\}$. If we let $s=\vee\left\{h_{n}(l), h_{n}(m), h_{n}(n)\right\}$, we can determine that $\frac{l}{(s, t)} \in h, \frac{m}{(s, t)} \in h$ and $\frac{n}{(s, t)} \in h$ but we know that $\frac{l p m q n}{(s, t)} \bar{\in}$ and $\frac{l p m q n}{(s, t)} \bar{q} h$. Thus, we obtain $\frac{l p m q n}{(s, t)} \overline{\in \vee q}$, which is impossible. Hence, $h_{n}(l p m q n) \leq \vee\left\{h_{n}(l), h_{n}(m), h_{n}(n),-0.5\right\}$ and $h_{p}($ lpmqn $) \geq \wedge\left\{h_{p}(l), h_{p}(m), h_{p}(n), 0.5\right\}$.

For the case (2) $\vee\left\{h_{n}(l), h_{n}(m), h_{n}(n)\right\} \leq-0.5$ and $\wedge\left\{h_{p}(l), h_{p}(m), h_{p}(n)\right\} \geq 0.5$. This implies $\frac{l}{(-0.5,0.5)} \in h, \frac{m}{(-0.5,0.5)} \in h$ and $\frac{n}{(-0.5,0.5)} \in h$ so $\frac{\text { lpmqn }}{(-0.5,0.5)} \in \vee q$. Thus, $\frac{\text { lpmqn }}{(-0.5,0.5)} \in h$ or $\frac{l p m q n}{(-0.5,0.5)} q h$. If $\frac{l p m q n}{(-0.5,0.5)} \in h$, we determine that $h_{n}(l p m q n) \leq-0.5 \leq \vee\left\{h_{n}(l), h_{n}(m), h_{n}(n),-0.5\right\}$ and $h_{p}(l p m q n) \geq 0.5 \geq \wedge\left\{h_{p}(l), h_{p}(m), h_{p}(n), 0.5\right\}$. If $\frac{l p m q n}{(-0.5,0.5)} q h$ we can determine that $h_{n}(l p m q n)-$ $0.5<-1$ and $h_{p}($ lpmqn $)+0.5>1$. This implies that $h_{n}($ lpmqn $)<-0.5$ and $h_{p}($ lpmqn $)>$ 0.5. Therefore, $h_{n}$ (lpmqn) $\leq-0.5 \leq \vee\left\{h_{n}(l), h_{n}(m), h_{n}(n),-0.5\right\}$ and $h_{p}($ lpmqn $) \geq 0.5 \geq$ $\wedge\left\{h_{p}(l), h_{p}(m), h_{p}(n), 0.5\right\}$.

For case (3), we have $h_{n}($ lpmqn $) \leq-0.5$ and $h_{p}($ lpmqn $) \geq \wedge\left\{h_{p}(l), h_{p}(m), h_{p}(n)\right\}$. Suppose that $h_{n}($ lpmqn $)>-0.5$ or $h_{p}($ lpmqn $)<\wedge\left\{h_{p}(l), h_{p}(m), h_{p}(n)\right\}$. If $h_{n}($ lpmqn $)>-0.5$, there exists $s \in[-1,0)$ such that $h_{n}($ lpmqn $)>s \geq-0.5 \geq \vee\left\{h_{n}(l), h_{n}(m), h_{n}(n)\right\}$. Let $t=\wedge\left\{h_{p}(l), h_{p}(m), h_{p}(n)\right\}$. This shows that $\frac{l}{(s, t)} \in h, \frac{m}{(s, t)} \in h$ and $\frac{n}{(s, t)} \in h$ but we know that $\frac{l p m q n}{(s, t)} \bar{\in}$ and $\frac{l p m q n}{(s, t)} \bar{q} h$. That is we obtain $\frac{(l p m q n)}{(s, t)} \in \vee q h$, which is impossible.

If $h_{p}($ lpmqn $)<\wedge\left\{h_{p}(l), h_{p}(m), h_{p}(n)\right\}$, we can determine that there exists $t \in(0,1]$ such that $h_{p}($ lpmqn $)<t \leq \wedge\left\{h_{p}(l), h_{p}(m), h_{p}(n)\right\}$. If we let $s=\vee\left\{h_{n}(l), h_{n}(m), h_{n}(n)\right\}$, we can determine that $\frac{l}{(s, t)} \in h, \frac{m}{(s, t)} \in h$ and $\frac{n}{(s, t)} \in h$ but we know that $\frac{l p m q n}{(s, t)} \bar{\in}$ and $\frac{l p m q n}{(s, t)} \bar{q} h$. That is, we obtain $\frac{(l p m q n)}{(s, t)} \overline{\in \vee q} h$, which is not possible. Hence, $h_{n}($ lpmqn $) \leq-0.5 \leq \vee\left\{h_{n}(l), h_{n}(m), h_{n}(n),-0.5\right\}$ and $h_{p}(l p m q n) \geq \wedge\left\{h_{p}(l), h_{p}(m), h_{p}(n)\right\} \geq \wedge\left\{h_{p}(l), h_{p}(m), h_{p}(n), 0.5\right\}$.

For case (4), we have $h_{n}($ lpmqn $) \leq \vee\left\{h_{n}(l), h_{n}(m), h_{n}(n)\right\}$ and $h_{p}($ lpmqn $) \geq 0.5$. Let $h_{n}($ lpmqn $)>\vee\left\{h_{n}(l), h_{n}(m), h_{n}(n)\right\}$ or $h_{p}($ lpmqn $)<0.5$. If $h_{n}(l p m q n)>\vee\left\{h_{n}(l), h_{n}(m), h_{n}(n)\right\}$, we can determine that there exists $s \in[-1,0)$ such that $h_{n}(l p m q n)>s \geq \vee\left\{h_{n}(l), h_{n}(m), h_{n}(n)\right\}$. Consequently, $\frac{l}{(s, 0.5)} \in h, \frac{m}{(s, 0.5)} \in h$ and $\frac{n}{(s, 0.5)} \in h$ but we know that $\frac{l p m q n}{(s, 0.5)} \bar{\in}$ and $\frac{l p m q n}{(s, 0.5)} \bar{q}$. That is, we obtain $\frac{l p m q n}{(s, 0.5)} \overline{\in V q}$, which is not possible. If $h_{p}($ lpmqn $)<0.5$, we can determine that there exists $t \in(0,1]$ with $h_{p}($ lpmqn $)<t \leq 0.5 \leq \wedge\left\{h_{p}(l), h_{p}(m), h_{p}(n)\right\}$. Let $s=\vee\left\{h_{n}(l), h_{n}(m), h_{n}(n)\right\}$. This implies that $\frac{l}{(s, t)} \in h, \frac{m}{(s, t)} \in h$ and $\frac{n}{(s, t)} \in h$ but we know that $\frac{l p m q n}{(s, t)} \bar{\in} h$ and $\frac{l p m q n}{(s, t)} \bar{q} h$. That is, we obtain $\frac{l p m q n}{(s, t)} \in \vee q$ which is a contradiction. Hence, $h_{n}($ lpmqn $) \leq \vee\left\{h_{n}(l), h_{n}(m), h_{n}(n)\right\} \leq$ $\vee\left\{h_{n}(l), h_{n}(m), h_{n}(n),-0.5\right\}$ and $h_{p}(l p m q n) \geq 0.5 \geq \wedge\left\{h_{p}(l), h_{p}(m), h_{p}(n), 0.5\right\}$.

The following example shows that the converse of the above theorem is not true in general. 
Example 3. Let $S=\{\alpha, \beta, \gamma, \delta, \varepsilon, \zeta\}$ be an ordered ternary semigroup defined by the following multiplication table and order:

$\begin{array}{lllllll}\cdot & \alpha & \beta & \gamma & \delta & \varepsilon & \zeta \\ \alpha & \alpha & \alpha & \alpha & \alpha & \alpha & \alpha \\ \beta & \alpha & \beta & \alpha & \delta & \varepsilon & \zeta \\ \gamma & \gamma & \gamma & \gamma & \gamma & \gamma & \gamma \\ \delta & \alpha & \delta & \alpha & \beta & \varepsilon & \delta \\ \varepsilon & \alpha & \varepsilon & \alpha & \varepsilon & \varepsilon & \varepsilon \\ \zeta & \alpha & \zeta & \alpha & \zeta & \varepsilon & \zeta\end{array}$

$\leq=\{(\alpha, \alpha),(\beta, \beta),(\gamma, \gamma),(\delta, \delta),(\varepsilon, \varepsilon),(\zeta, \zeta),(\zeta, \varepsilon)\}$. Let a bipolar fuzzy set $h=\left(S ; h_{n}, h_{p}\right)$ be defined as:

$$
\begin{array}{ccccccc}
S & \alpha & \beta & \gamma & \delta & \varepsilon & \zeta \\
h_{n} & -0.10 & -0.3 & -0.9 & -0.4 & -0.5 & -0.7 \\
h_{p} & 0.8 & 0.3 & 0.7 & 0.4 & 0.4 & 0.5
\end{array}
$$

By simple calculations, we can prove that $h$ satisfies the conditions (1) and (2) of the above theorem. However, $h$ is not an $(\in, \in \vee q)$-bipolar fuzzy generalized bi-ideal of $S$, since $\frac{\beta}{(-0.28,0.38)} \bar{\in} h$ because $h_{p}(\beta)=0.3<0.38$. Furthermore, $h_{n}(\beta)+(-0.28)=-0.2-0.28=-0.48<-1$ and $h_{p}(\beta)+(0.38)=0.3+0.38=0.68>1$ implies that $\frac{\beta}{(-0.28,0.38)} \bar{q} h$. Therefore, $\frac{\beta}{(-0.28,0.38)} \overline{\in V q} h$.

It is very easy to determine that every $(\in, \in \vee q)$-bipolar fuzzy bi-ideal is an $(\in, \in \vee q)$-bipolar fuzzy generalized bi-ideal but the converse is not true in general.

Example 4. Suppose that we consider $S$ as an ordered ternary semigroup given in Example 1 and we define bipolar fuzzy set as follows:

$$
\begin{array}{ccccc}
S & \alpha & \beta & \gamma & \delta \\
h_{n} & -0.8 & -0.4 & -0.6 & -0.2 \\
h_{p} & 0.8 & 0.2 & 0.4 & 0
\end{array}
$$

Thus, $h=\left(S ; h_{n}, h_{p}\right)$ is evidently an $(\in, \in \vee q)$-bipolar fuzzy generalized bi-ideal of $S$ for all $l, m \in S$ such that: We have $h_{n}(l) \leq h_{n}(m)$ and $h_{p}(l) \geq h_{p}(m)$. Furthermore, $h_{n}($ lpmqn $)=h_{n}(a)=-0.7 \leq$ $\vee\left\{h_{n}(l), h_{n}(m), h_{n}(n),-0.5\right\}$ and $h_{p}(l p m q n)=h_{p}(\alpha)=0.8 \geq \wedge\left\{h_{p}(l), h_{p}(m), h_{p}(n), 0.5\right\}$ for all $l, m, n, p, q \in S$. However, $h_{n}(\gamma \cdot \gamma \cdot \gamma)=h_{n}(\beta)=-0.3>-0.5=\vee\left\{h_{n}(\gamma), h_{n}(\gamma), h_{n}(\gamma),-0.5\right\}$ and $h_{p}(\gamma \cdot \gamma \cdot \gamma)=h_{p}(\beta)=0.2<0.4=\vee\left\{h_{p}(\gamma), h_{p}(\gamma), h_{p}(\gamma), 0.5\right\}$. Hence, $h=\left(S ; h_{n}, h_{p}\right)$ is not an $(\in, \in \vee q)$-bipolar fuzzy bi-ideal of $S$.

Theorem 9. Let $h=\left(S ; h_{n}, h_{p}\right)$ be a non-zero $(\in, \in \vee q)$-bipolar fuzzy generalized bi-ideal of $S$. Thus, the set $S_{\circ}=\left\{l \in S \mid h_{n}(l) \neq 0\right\} \cap\left\{l \in S \mid h_{p}(l) \neq 0\right\}$ is a generalized bi-ideal of $S$.

Proof. Let $h=\left(S ; h_{n}, h_{p}\right)$ be a non-zero $(\in, \in \vee q)$-bipolar fuzzy generalized bi-ideal of $S$. Let $l, m \in S$ such that $l \leq m$ and $m \in S_{\circ}$. Thus, $h_{n}(m) \neq 0$ and $h_{p}(m) \neq 0$, so $h_{n}(m)<0$ and $h_{p}(m)>0$. Suppose $h_{n}(l)=0$ or $h_{p}(l)=0$. Since $\frac{m}{\left(h_{n}(m), h_{p}(m)\right)} \in h$ and $h_{n}(l)>h_{n}(m)$ or $h_{p}(l)<h_{p}(m)$. It follows $\frac{l}{\left(h_{n}(m), h_{p}(m)\right)} \bar{\in}$, which shows a contradiction. Furthermore, $h_{n}(l)+h_{n}(m)=h_{n}(m) \geq-1$ or $h_{p}(l)+h_{p}(m)=h_{p}(m) \leq 1$. Thus, $h_{n}(l)+h_{n}(m) \geq-1$ or $h_{p}(l)+h_{p}(m) \leq 1$. This implies $\frac{l}{\left(h_{n}(l)+h_{n}(m)\right)} \bar{q} h$, which contradicts the statement. Therefore, $h_{n}(l) \neq 0$ and $h_{p}(l) \neq 0$. Thus, $l \in S_{\circ}$. Let $l, m, n, p, q \in S$ such that $l, m, n \in S_{\circ}$. Thus, $h_{n}(l) \neq 0, h_{n}(m) \neq 0, h_{n}(n) \neq 0$, $h_{p}(l) \neq 0, h_{p}(m) \neq 0$ and $h_{p}(n) \neq 0$. Thus, $h_{n}(l)<0, h_{n}(m)<0, h_{n}(n)<0, h_{p}(l)>0$, $h_{p}(m)>0$ and $h_{p}(n)>0$. If we suppose that lpmqn $\notin S_{\circ}$, we know that $h_{n}($ lpmqn) $=0$ or $h_{p}(l p m q n)=0$. Clearly, $\frac{l}{\left(h_{n}(l), h_{p}(l)\right)} \in h, \frac{m}{\left(h_{n}(m), h_{p}(m)\right)} \in h$ and $\frac{n}{\left(h_{n}(n), h_{p}(n)\right)} \in h$. Since $h_{n}(l p m q n)=$ $0>\vee\left\{h_{n}(l), h_{n}(m), h_{n}(n)\right\}$ or $h_{p}($ lpmqn $)=0<\wedge\left\{h_{p}(l), h_{p}(m), h_{p}(n)\right\}$. Thus, $h_{p}($ lpmqn $)<$ $\wedge\left\{h_{p}(l), h_{p}(m), h_{p}(n)\right\}$, which implies $\frac{l p m q n}{\left(\vee\left\{h_{n}(l), h_{n}(m), h_{n}(n)\right\}, \wedge\left\{h_{p}(l), h_{p}(m), h_{p}(n)\right\}\right)} \bar{\in} h$. This is a contradiction. 
Furthermore, $h_{n}($ lpmqn $)+\vee\left\{h_{n}(l), h_{n}(m), h_{n}(n)\right\}=\vee\left\{h_{n}(l), h_{n}(m), h_{n}(n)\right\} \geq-1$ or $h_{p}($ lpmqn $)+$ $\wedge\left\{h_{p}(l), h_{p}(m), h_{p}(n)\right\}=\wedge\left\{h_{p}(l), h_{p}(m), h_{p}(n) \leq 1\right.$. Thus, $h_{p}($ lpmqn $)+\wedge\left\{h_{p}(l), h_{p}(m), h_{p}(n) \leq 1\right.$. This implies that $\frac{l p m q n}{\left(\vee\left\{h_{n}(l), h_{n}(m), h_{n}(n)\right\} \wedge\left\{h_{p}(l), h_{p}(m), h_{p}(n)\right\}\right)} \bar{q} h$, which is a contradiction. Therefore, $h_{n}($ lpmqn $) \neq 0$ and $h_{p}(l p m q n) \neq 0$. Hence, lpmqn $\in S_{\circ}$. Thus, $S_{\circ} S S_{\circ} S S_{\circ} \subseteq S_{\circ}$.

Theorem 10. If $h=\left(S ; h_{n}, h_{p}\right)$ is a non-zero $(q, \in \vee q)$-bipolar fuzzy generalized bi-ideal of $S$. Thus, the set $S_{\circ}=\left\{l \in S \mid h_{n}(l) \neq 0\right\} \cap\left\{l \in S \mid h_{p}(l) \neq 0\right\}$ is a generalized bi-ideal of $S$

Proof. Let $h=\left(S ; h_{n}, h_{p}\right)$ be a non-zero $(q, \in \vee q)$-bipolar fuzzy generalized bi-ideal of $S$. Let $l, m \in S$, such that $l \leq m$ with $m \in S_{\circ}$. This implies that $h_{n}(m) \neq 0$ and $h_{p}(m) \neq 0$. Thus, $h_{n}(m)<0$ and $h_{p}(m)>0$ according to Definition 5. Hence, $h_{n}(m)-1<-1$ and $h_{p}(m)+1>1$. This implies $\frac{m}{(-1,1)} q h$. Suppose if $h_{n}(l)=0$ or $h_{p}(l)=0$. Thus, $h_{n}(l)>-1$ or $h_{p}(l)<1$, so $\frac{l}{(-1,1)} \bar{\epsilon}$, which contradicts Definition 5. Therefore, $h_{n}(l) \neq 0$ and $h_{p}(l) \neq 0$. Hence, $l \in S_{\circ}$. Let $l, m, n, p, q \in S$ such that $l, m, n \in$ $S_{\circ}$. Thus, $h_{n}(l) \neq 0, h_{n}(m) \neq 0, h_{n}(n) \neq 0, h_{p}(l) \neq 0, h_{p}(m) \neq 0$ and $h_{p}(n) \neq 0$. This represents $h_{n}(l)<0, h_{n}(m)<0, h_{n}(n)<0, h_{p}(l)>0, h_{p}(m)>0$ and $h_{p}(n)>0$. Hence, $h_{n}(l)-1<-1, h_{n}(m)-$ $1<-1, h_{n}(n)-1<-1, h_{p}(l)+1<1, h_{p}(m)+1<1$, and $h_{p}(n)+1<1$. It follows that $\frac{l}{(-1,1)} q h$, $\frac{m}{(-1,1)} q h$ and $\frac{n}{(-1,1)} q h$. If $h_{n}($ lpmqn $)=0$ or $h_{p}($ lpmqn $)=0$ we can determine that $h_{n}($ lpmqn $)>-1$ or $h_{p}(l p m q n)<1$. This implies that $\frac{l p m q n}{(-1,1)} \bar{\in}$, which is not possible. Furthermore, $h_{n}(l p m q n)-1=-1$ or $h_{p}($ lpmqn $)+1=1$. This shows that $\frac{l p m q n}{(-1,1)} \bar{q} h$, which contradicts the supposition. Therefore, $h_{n}(l p m q n) \neq 0$ and $h_{p}(l p m q n) \neq 0$. Hence, lpmqn $\in S_{\circ}$. Thus, $S_{\circ} S S_{\circ} S S_{\circ} \subseteq S_{\circ}$. Hence, $S_{\circ}$ is a generalized bi-ideal of $S$.

\section{Upper and Lower Parts of $(\in, \in \vee q)$-Bipolar Fuzzy Generalized Bi-Ideals}

In this section, we have studied the upper and lower parts of the $(\in, \in \vee q q)$ bipolar fuzzy generalized bi-ideal in ordered ternary semigroups and obtained several interesting results. Furthermore, in this section, we have studied the regularity of the ordered ternary semigroups and have proved various results.

The proofs of the following Lemmas are obvious.

Lemma 3. Let $u=\left(S ; u_{n}, u_{p}\right), v=\left(S ; v_{n}, v_{p}\right)$ and $w=\left(S ; w_{n}, w_{p}\right)$ be bipolar fuzzy sets in $S$. Thus, the following equations are satisfied:

1. $(u \wedge v)^{-}=\left(u^{-} \wedge v^{-}\right)$

2. $(u \vee v)^{-}=\left(u^{-} \vee v^{-}\right)$

3. $(u \circ v \circ w)^{-}=\left(u^{-} \circ v^{-} \circ w^{-}\right)$.

Lemma 4. Let $u=\left(S ; u_{n}, u_{p}\right), v=\left(S ; v_{n}, v_{p}\right)$ and $w=\left(S ; w_{n}, w_{p}\right)$ be bipolar fuzzy sets in $S$. Thus, the following equations are satisfied:

1. $(u \wedge v)^{+}=\left(u^{+} \wedge v^{+}\right)$

2. $(u \vee v)^{+}=\left(u^{+} \vee v^{+}\right)$

3. $(u \circ v \circ w)^{+} \geq\left(u^{+} \circ v^{+} \circ w^{+}\right)$if $F_{a}=\varphi$.

Lemma 5. If $F, G$ and $H$ represents the non-empty subsets of $S$ the following equations are satisfied:

1. $F \subseteq G$ if and only if $\chi_{F}^{-} \subseteq \chi_{G}^{-}$

2. $\left(\chi_{F} \wedge \chi_{G}\right)^{-}=\chi_{F \cap G}^{-}$

3. $\left(\chi_{F} \vee \chi_{G}\right)^{-}=\chi_{F \cup G}^{-}$

4. $\left(\chi_{F} \circ \chi_{G} \circ \chi_{H}\right)^{-}=\chi_{(F G H]}^{-}$.

Proof. The proofs of (1), (2) and (3) are quite clear. 
(4) Consider $k \in(F G H]$. Thus, $\chi_{n(F G H]}^{-}(k)=-0.5$ and $\chi_{p(F G H]}^{-}(k)=0.5$. As $k \in(F G H]$ with $k \leq l m n$ for some $l \in F$, we can determine that $m \in G$ and $n \in H$. Consequently, $(l, m, n) \in A_{k}$ with $A_{k} \neq \varphi$. Since $l \in F, m \in G$ and $n \in H$ so $\chi_{n F}(l)=-1, \chi_{p F}(l)=1, \chi_{n G}(m)=-1, \chi_{p G}(m)=1$ $\chi_{n H}(n)=-1$ and $\chi_{p H}(n)=1$. Thus, we can determine:

$$
\begin{aligned}
\left(\chi_{n F} \circ \chi_{n G} \circ \chi_{n H}\right)^{-}(k)= & \left(\chi_{n F} \circ \chi_{n G} \circ \chi_{n H}\right)(k) \vee-0.5 \\
& =\hat{(p, q, r) \in A_{k}}\left\{\left(\chi_{n F}(p) \vee \chi_{n G}(q) \vee \chi_{n H}(r)\right)\right\} \vee-0.5 \\
& \leq\left\{\chi_{n F}(l) \vee \chi_{n G}(m) \vee \chi_{n H}(n)\right\} \vee-0.5 \\
& =(-1 \vee-1 \vee-1) \vee-0.5 \\
& =-0.5=\chi_{n(F G H]}^{-}(k) .
\end{aligned}
$$

Furthermore, we obtain:

$$
\begin{aligned}
\left(\chi_{p F} \circ \chi_{p G} \circ \chi_{p H}\right)^{-}(k) & =\left(\chi_{p F} \circ \chi_{p G} \circ \chi_{p H}\right)^{-}(k) \wedge 0.5 \\
& \left.=\vee \vee \vee \chi_{p F}(p) \wedge \chi_{p G}(q) \wedge \chi_{p H}(r)\right\} \wedge 0.5 \\
& \geq\left\{\chi_{p F}(l) \wedge \chi_{p G}(m) \wedge \chi_{p H}(n)\right\} \wedge 0.5 \\
& =(1 \wedge 1 \wedge 1) \wedge 0.5 \\
& =0.5=\chi_{p(F G H]}^{-}(k) .
\end{aligned}
$$

If we let $k \notin(F G H]$, we can determine that $\chi_{n(F G H]}^{-}(k)=0$ and $\chi_{p(F G H]}^{-}(k)=0$. Thus, $\left(\chi_{n F} \circ \chi_{n G} \circ \chi_{n H}\right)^{-}(k)=\left(\chi_{n F} \circ \chi_{n G} \circ \chi_{n H}\right)(k) \vee-0.5=0 \vee-0.5=0=\chi_{n(F G H]}^{-}(k)$ and $\left(\chi_{p F} \circ \chi_{p G} \circ \chi_{p H}\right)^{-}(k)=\left(\chi_{p F} \circ \chi_{p G} \circ \chi_{p H}\right)(k) \wedge 0=0=\chi_{p(F G H]}^{-}(k)$. That is, $\left(\chi_{F} \circ \chi_{G} \circ \chi_{H}\right)^{-}=\chi_{(F G H]}^{-}$.

Lemma 6. If a subset $F$ is the generalized bi-ideal of $S$, we can determine that $\chi_{F}^{-}=\left(S ; \chi_{n F}^{-}, \chi_{p F}^{-}\right)$is an $(\in, \in \vee q)$-bipolar fuzzy generalized bi-ideal of $S$.

Lemma 7. If a subset $F$ is a left (resp. right, lateral) ideal of $S$, we can determine that $\chi_{F}^{-}=\left(S ; \chi_{n F}^{-}, \chi_{p F}^{-}\right)$is an $(\in, \in \vee q)$-bipolar fuzzy left(resp. right, lateral) ideal of $S$.

Proposition 1. If we let $h=\left(S ; h_{n}, h_{p}\right)$ be an $(\in, \in \vee q)$-bipolar fuzzy generalized bi-ideal of $S_{\text {, }}$ we can determine that $h^{-}=\left(S ; h_{n}^{-}, h_{p}^{-}\right)$is a bipolar fuzzy generalized bi-ideal of $S$.

Proof. Let that $h^{-}=\left(S ; h_{n}^{-}, h_{p}^{-}\right)$is an $(\in, \in \vee q)$-bipolar fuzzy generalized bi-ideal of $S$. Suppose $l, m \in S$ such that $l \leq m$. Thus, $h_{n}(l) \leq h_{n}(m) \vee-0.5$ and $h_{p}(l) \geq h_{p}(m) \wedge 0.5$. Therefore, $h_{n}(l) \vee$ $-0.5 \leq\left(h_{n}(m) \vee-0.5\right) \vee-0.5=h_{n}(m) \vee-0.5$ and $h_{p}(l) \wedge 0.5 \geq\left(h_{p}(m) \wedge 0.5\right) \wedge 0.5=h_{p}(m) \wedge 0.5$. This implies that $h_{n}^{-}(l) \leq h_{n}^{-}(m)$ and $h_{p}^{-}(l) \geq h_{p}^{-}(m)$.

If we let $p, q, l, m, n \in S$, we can determine that $h_{n}($ lpmqn $) \leq\left(h_{n}(l) \vee h_{n}(m) \vee h_{n}(n)\right) \vee-0.5$ and $h_{p}($ lpmqn $) \geq\left(h_{p}(l) \wedge h_{p}(m) \wedge h_{p}(n)\right) \wedge-0.5$ by using the second condition of Theorem 15 . Thus,

$h_{n}($ lpmqn $) \vee-0.5 \leq\left(h_{n}(l) \vee h_{n}(m) \vee h_{n}(n)\right) \vee-0.5$. This shows that $h_{n}($ lpmqn $) \vee-0.5 \leq$ $\left(h_{n}(l) \vee-0.5\right) \vee\left(h_{n}(m) \vee-0.5\right) \vee\left(h_{n}(n) \vee-0.5\right)$. This becomes $h_{n}^{-}($lpmqn $) \leq h_{n}^{-}(l) \vee h_{n}^{-}(m) \vee h_{n}^{-}(n)$ and $h_{p}^{-}($lpmqn $) \wedge 0.5 \geq\left(h_{p}^{-}(l) \wedge 0.5\right) \wedge\left(h_{p}^{-}(m) \wedge 0.5\right) \wedge\left(h_{p}^{-}(n) \wedge 0.5\right)$. This implies that $h_{p}^{-}($lpmqn $) \geq$ $h_{p}^{-}(l) \wedge h_{p}^{-}(m) \wedge h_{p}^{-}(n)$. Thus, $h^{-}=\left(S ; h_{n}^{-}, h_{p}^{-}\right)$is a bipolar fuzzy generalized bi-ideal of $S$.

Now, for the next theorems, we will study the regular ordered ternary semigroups in the form of the lower parts of $(\in, \in \vee q)$-bipolar fuzzy left (resp. right or two-sided) ideals and $(\in, \in \vee q)$-bipolar fuzzy generalized bi-ideals. An ordered ternary semigroup $S$ is called regular if $a \in(a S a]$ for any $a \in S$ [21]. 
Theorem 11. Any $S$ is regular if and only if each $(\in, \in \vee q)$-bipolar fuzzy generalized bi-ideal $h=\left(S ; h_{n}, h_{p}\right)$ and each $(\in, \in \vee q)$-bipolar fuzzy left ideal $g=\left(S ; g_{n}, g_{p}\right)$ of $S$. Thus, it becomes $(h \wedge g)^{-} \leq(h \circ 1 \circ g)^{-}$.

Proof. Let $(h \wedge g)^{-} \leq(h \circ 1 \circ g)^{-}$for each $(\in, \in \vee q)$-bipolar fuzzy generalized bi-ideal $h=\left(S, h_{n}, h_{p}\right)$, each $(\in, \in \vee q)$-bipolar fuzzy left ideal $g=\left(S ; g_{n}, g_{p}\right)$ of $S$. We need to show that $S$ is regular. Using Lemma 2, we only show that $F \cap L \subseteq(F S L]$ for each generalized bi-ideal $F$ and each left ideal $L$ of $S$. Consider $u \in F \cap L$. This shows that $u \in F, u \in L$. Using Lemma 22 and 23, we get $\chi_{F}^{-}$as an $(\in, \in \vee q)$-bipolar fuzzy generalized bi-ideal and $\chi_{L}^{-}$as an $(\in, \in \vee q)$-bipolar fuzzy left ideal of $S$. Using this assumption, $\chi_{[F S L]}^{-}(u)=\chi_{F}^{-}(u) \circ \chi_{S}^{-}(u) \circ \chi_{L}^{-}(u) \geq \chi_{F}^{-}(u) \wedge \chi_{S}^{-}(u) \wedge \chi_{L}^{-}(u)=\chi_{F \cap S \cap L}^{-}(u)$ by using Definition 5. Therefore, $F \cap S \cap L \subseteq(F S L]$ and $S$ is regular.

Conversely, suppose that $S$ is regular and $u \in S$. Thus, there exists $l \in S$ such that $u \leq u l u \leq$ $u l(u l u) \leq(u l u l u) l(u l u)$. This shows that $(u l u l u, l, u l u) \in A_{u}$ so $A_{u} \neq \varphi$. Now, we obtain:

$$
\begin{aligned}
\left(h_{n} \circ 1_{n} \circ g_{n}\right)^{-}(u) & =\left(h_{n} \circ 1_{n} \circ g_{n}\right)(u) \vee-0.5 \\
& =\hat{\wedge}\left\{\left(h_{n}\right)(l) \vee 1_{n}(m) \vee g_{n}(n)\right\} \vee-0.5 \\
& \leq\left\{\left(h_{n}\right)(\text { ululu }) \vee 1_{n}(l) \vee g_{n}(u l u)\right\} \vee-0.5
\end{aligned}
$$

Since $h=\left(S, h_{n}, h_{p}\right)$ is a $(\in, \in \vee q)$-bipolar fuzzy generalized bi-ideal of $S$, so $\left(h_{n}\right)(u l u l u) \leq\left(h_{n}\right)(u) \vee$ $h_{n}(u) \vee h_{n}(u) \vee-0.5=\left(h_{n}\right)^{-}(u)$ and $g=\left(S ; g_{n}, g_{p}\right)$ is $(\in, \in \vee q)$-bipolar fuzzy left ideal of $S$. Thus, $g_{n}(u l u) \leq g_{n}(u) \vee-0.5=g_{n}^{-}(u)$. Hence, $\left(h_{n} \circ 1_{n} \circ g_{n}\right)^{-}(u) \leq\left(h_{n} \wedge g_{n}\right)^{-}(u)$. Similarly, we can prove that $\left(h_{p} \circ 1_{p} \circ g_{p}\right)^{-}(u) \geq\left(h_{p} \wedge g_{p}\right)^{-}(u)$. Thus, we have the required result of $(h \wedge g)^{-} \leq(h \circ 1 \circ g)^{-}$.

Theorem 12. Any $S$ is regular if and only if each $(\in, \in \vee q)$-bipolar fuzzy generalized bi-ideal $h=\left(S ; h_{n}, h_{p}\right)$ and each $(\in, \in \vee q)$-bipolar fuzzy ideal $g=\left(S ; g_{n}, g_{p}\right)$ of $S$. Thus, it becomes $(h \wedge g)^{-} \leq(h \circ g \circ h)^{-}$.

Proof. Let $(h \wedge g)^{-} \leq(h \circ g \circ h)^{-}$for each $(\in, \in \vee q)$-bipolar fuzzy generalized bi-ideal $h=\left(S ; h_{n}, h_{p}\right)$ and each $(\in, \in \vee q)$-bipolar fuzzy ideal $g=\left(S ; g_{n}, g_{p}\right)$ of $S$. Now, to prove that $S$ is regular, we just need to show that $F \cap I \subseteq(F I F]$ for each ideal $I$ and every generalized bi-ideal $F$ of $S$ by Lemma 1 . Let $b \in F \cap I$ at that instant $b \in I$ and $b \in F$. Thus, Lemma 22 shows that $\chi_{F}^{-}=\left(S ; \chi_{n F}^{-}, \chi_{p F}^{-}\right)$ is an $(\in, \in \vee q)$-bipolar fuzzy generalized bi-ideal of $S$ and through Lemma $23, \chi_{I}^{-}=\left(S ; \chi_{n I}^{-}, \chi_{p I}^{-}\right)$ is an $(\in, \in \vee q)$-bipolar fuzzy ideal of $S$. Thus, through the given supposition, we get $\chi_{[F I F]}^{-}(b)=$ $\chi_{F}^{-}(b) \circ \chi_{I}^{-}(b) \circ \chi_{F}^{-}(b) \geq \chi_{F}^{-}(b) \wedge \chi_{I}^{-}(b)=\chi_{F \cap I}^{-}(b)$. This shows $\chi_{(F I F]}^{-}(b) \geq \chi_{F \cap I}^{-}(b)$ by using Lemma 5 . Therefore, $F \cap I \subseteq(F I F]$. This proves that $S$ is regular.

On contrary, assume that $S$ is regular with $b \in S$. Thus, there exists $l \in S$ such that $b \leq b l b \leq$ $\left(b l(b l b)\right.$. Thus, $(b,(l b l), b) \in A_{b} \neq \varphi$. Now, we obtain

$$
\begin{aligned}
\left(h_{n} \circ g_{n} \circ h_{n}\right)^{-}(b) & =\left(h_{n} \circ g_{n} \circ h_{n}\right)(b) \vee-0.5 \\
& =\bigwedge_{(l, m, n) \in A_{a}}^{\wedge}\left(h_{n} \circ g_{n} \circ h_{n}\right)(b) \vee-0.5 \\
& =\bigwedge_{(l, m, n) \in A_{b}}^{\wedge}\left\{h_{n}(l) \vee g_{n}(m) \vee h_{n}(n)\right\} \vee-0.5 \\
& \leq\left\{h_{n}(b) \vee g_{n}(l b l) \vee h_{n}(b)\right\} \vee-0.5 .
\end{aligned}
$$

Since $g=\left(S ; g_{n}, g_{p}\right)$ is an $(\in, \in \vee q)$-bipolar fuzzy ideal of $S$. Thus, $g_{n}(l b l) \leq g_{n}(b) \vee-0.5$. Thus, we have

$$
\begin{aligned}
\left\{h_{n}(b) \vee g_{n}(l b l) \vee h_{n}(b)\right\} \vee-0.5 & \leq\left\{h_{n}(b) \vee-0.5\right\} \vee\left\{g_{n}(b) \vee-0.5\right\} \vee\left\{h_{n}(b) \vee-0.5\right\} \\
& =h_{n}^{-}(b) \vee g_{n}^{-}(b) \vee h_{n}^{-}(a) \\
& \leq\left(h_{n}^{-} \vee g_{n}^{-} \vee h_{n}^{-}\right)(b)
\end{aligned}
$$


In a same way, we can easily show that $\left(h_{p} \circ g_{p} \circ h_{p}\right)^{-}(b) \geq\left(h_{p} \wedge g_{p}\right)^{-}(b)$. Hence, $\left(h_{p} \wedge g_{p}\right)^{-} \leq$ $\left(h_{p} \circ g_{p} \circ h_{p}\right)^{-}$

Theorem 13. Any $S$ is regular if and only if each $(\in, \in \vee q)$-bipolar fuzzy right ideal $h=\left(S, h_{n}, h_{p}\right)$, each $(\in, \in \vee q)$-bipolar fuzzy generalized bi-ideal $g=\left(S ; g_{n}, g_{p}\right)$ and each $(\in, \in \vee q)$-bipolar fuzzy left ideal $f=\left(S ; f_{n}, f_{p}\right)$ of $S$. Thus, we get $(h \wedge g \wedge f)^{-} \leq(h \circ 1 \circ g \circ 1 \circ f)^{-}$

Proof. Let $(h \wedge g \wedge f)^{-} \leq(h \circ g \circ f)^{-}$for each $(\in, \in \vee q)$-bipolar fuzzy right ideal $h=\left(S ; h_{n}, h_{p}\right)$, each $(\in, \in \vee q)$-bipolar fuzzy generalized bi-ideal $g=\left(S ; g_{n}, g_{p}\right)$ and each $(\in, \in \vee q)$-bipolar fuzzy left ideal $f=\left(S ; f_{n}, f_{p}\right)$ of $S$. We have to show that $S$ is regular. Using Lemma 2 , we only show that $R \cap F \cap L \subseteq(R S F S L]$ for each right ideal $R$, each generalized bi-ideal $F$ and each left ideal $L$ of $S$. Consider $u \in R \cap F \cap L$ Using Lemmas 22 and 23 , we get $\chi_{R}^{-}=\left(S ; \chi_{n R}^{-}, \chi_{p R}^{-}\right)$as an $(\in, \epsilon$ $\vee q)$-bipolar fuzzy right ideal $\chi_{F}^{-}=\left(S ; \chi_{n F}^{-}, \chi_{p F}^{-}\right)$as an $(\in, \in \vee q)$-bipolar fuzzy generalized bi-ideal and $\chi_{L}^{-}=\left(S ; \chi_{n L}^{-}, \chi_{p L}^{-}\right)$as an $(\in, \in \vee q)$-bipolar fuzzy left ideal of $S$. Using the assumption, $\chi_{(R F L]}^{-}(u)=$ $\chi_{R}^{-}(u) \circ \chi_{F}^{-}(u) \circ \chi_{L}^{-}(u) \geq \chi_{R}^{-}(u) \wedge \chi_{F}^{-}(u) \wedge \chi_{L}^{-}(u)=\chi_{R \cap F \cap L}^{-}(u)$ by using Definition 5. Therefore, $R \cap F \cap L \subseteq(R S F S L]$ and $S$ is regular.

Conversely, suppose that $S$ is regular and $u \in S$. Thus, there exists $l \in S$ such as $u \leq u l u \leq u l(u l u)$. This shows that $(u, l, u l u),(u l u, l, u) \in A_{u}$ so $A_{u} \neq \varphi$ and

$$
\begin{aligned}
\left(h_{n} \circ 1_{n} \circ g_{n} \circ 1_{n} \circ f_{n}\right)^{-}(u) & =\left(h_{n} \circ 1_{n} \circ g_{n} \circ 1_{n} \circ f_{n}\right)(u) \vee-0.5 \\
& =\left(u l \wedge \hat{(u l u, l, u) \in A_{u}}\left\{h_{n}(u l u) \vee 1_{n}(l) \vee\left(g_{n} \circ 1_{n} \circ f_{n}\right)(u)\right\} \vee-0.5\right. \\
& \leq \min \left[h_{n}(u) \underset{(p, t, q) \in A_{u}}{\vee}\left\{g_{n}(p) \vee 1_{n}(t) \vee f_{n}(q)\right\} \vee-0.5\right. \\
& \leq \min \left[h_{n}(u) \min \left\{g_{n}(u) \vee 1_{n}(l) \vee f_{n}(u l u)\right\}\right] \vee-0.5 \\
& =\min \left[h_{n}(u) \min \left\{g_{n}(u), f_{n}(u l u)\right\}\right] \vee-0.5 \\
& \leq \min \left[h_{n}(u) \min \left\{g_{n}(u), f_{n}(u)\right\}\right] \vee(-0.5) \\
& =\min \left\{h_{n}(u), g_{n}(u), f_{n}(u)\right\} \vee(-0.5) \\
& =\left(h_{n} \wedge g_{n} \wedge f_{n}\right)^{-}(u)
\end{aligned}
$$

In a similar way, we can easily show that $\left(h_{p} \circ 1_{p} \circ g_{p} \circ 1_{p} \circ f_{p}\right)^{-}(u) \geq\left(h_{p} \wedge g_{p} \wedge f_{p}\right)^{-}(u)$. Hence, we get $(h \wedge g \wedge f)^{-} \leq(h \circ 1 \circ g \circ 1 \circ f)^{-}$, which is the required result.

\section{Conclusions}

The ternary ordered semigroups are a joined extension of ordered semigroups and ternary semigroups. Similar to the case of ordered semigroups, the bipolar fuzzy subsets of an ordered ternary semigroup is proved to be an ordered ternary semigroup. In addition, we have proved that if a bipolar fuzzy set $h=\left(S, h_{n}, h_{p}\right)$ in an ordered ternary semigroup $S$ is $(\in, \in \vee q)$-bipolar fuzzy generalized bi-ideal of $S$, we can determine that it satisfies two particular properties of $S$ but we have shown with an example that the reverse of this statement is not true in general. This major result has negated the result proved in [20]. A technique of bipolar fuzzification is applied to study the regular ordered ternary semigroups.

Our future plans are to apply this technique for gamma semigroup and near rings. Thus, we will further generalize it by defining the roughness of bipolar fuzzy ideals of ternary semigroups, gamma semigroups and near-rings.

Author Contributions: Conceptualization: S.B. and M.S.; Methodology: M.F.; Software: S.B.; Validation: M.F., S.B.; Formal Analysis: M.F.; Investigation: M.S.; Resources: S.B.; Data Curation, M.S.; Writing-Original Draft Preparation: M.F.; Writing-Review and Editing: S.B.; Visualization: M.F.; Supervision: S.B.; Project Administration: M.F.

Funding: This research was funded by University of Gujrat, Gujrat, Pakistan. 
Conflicts of Interest: The authors declare that they have no conflict of interest.

\section{References}

1. Zadeh, L.A. Fuzzy sets. Inf. Control 1965, 8, 338-353. [CrossRef]

2. Zhang, W.R. Bipolar fuzzy sets and relations: A computational framework for cognitive modeling and multiagent decision analysis. In Proceedings of the Industrial Fuzzy Control and Intelligent Systems Conference, and the NASA Joint Technology Workshop on Neural Networks and Fuzzy Logic and Fuzzy Information Processing Society Biannual Conference, San Antonio, TX, USA, 18-21 December 1994; pp. 305-309.

3. Ibrar, M.; Davaaz, B.; Khan, A. Characterizations of regular ordered semigroups in terms of $(\alpha, \beta)$-fuzzy generalized bi-ideals. Inf. Sci. 2011, 181, 1759-1770.

4. Jun, Y.B.; Kavikumar, J. Bipolar fuzzy finite state machines. Bull. Malays. Math. Sci. Soc. 2011, 34, 181-188.

5. Zhou, M.; Li, S. Applications of bipolar fuzzy theory to hemirings. Int. J. Innov. Comput. Inf. Control 2013, 199, 1349-4198.

6. Zhou, M.; Li, S. Applications of bipolar fuzzy theory in semirings. J. Math. Res. Appl. 2014, 34, 61-72.

7. Jun, Y.B.; Meng, J.; Xin, X.L. On ordered filters of implicative semigroups. Semigroup Forum 1997, 54, 75-82. [CrossRef]

8. Kehayopulu, N. On weakly prime ideals of ordered semigroups. Semigroup Forum 1992, 44, 341-346. [CrossRef]

9. Kehayopulu, N. On intra-regular ordered semigroups. Semigroup Forum 1993, 46, 271-278. [CrossRef]

10. Kehayopulu, N.; Tsingelis, M. Fuzzy sets in ordered groupoids. Semigroup Forum 2002, 65, 128-132. [CrossRef]

11. Kehayopulu, N.; Tsingelis, M. The embedding of an ordered groupoid in to a poe-groupoid in terms of fuzzy sets. Inf. Sci. 2003, 152, 231-236. [CrossRef]

12. Kehayopulu, N.; Tsingelis, M. Intra-regular ordered semigroup in terms of fuzzy sets. Lobachevskii J. Math. 2009, 30, 23-29. [CrossRef]

13. Kehayopulu, N. Left quasi-regular and intra-regular ordered semigroups using fuzzy ideals. Quasigroups Relat. Syst. 2012, 20, 239-249.

14. Shabir, M.; Khan, A. On fuzzy ordered semigroups. Inf. Sci. 2014, 274, 236-248. [CrossRef]

15. Shabir, M.; Khan, A. Characterizations of ordered semigroups by the properties of their fuzzy ideals. Comput. Math. Appl. 2010, 59, 539-549. [CrossRef]

16. Shabir, M.; Khan, A. Characterizations of ordered semigroups by the properties of their fuzzy generalized bi-ideals. New Math. Nat. Comput. 2008, 4, 237-250. [CrossRef]

17. Daddi, V.R.; Pawar, Y.S. On ordered ternary semigroups. Kyungpook Math. J. 2012, 52, 375-381. [CrossRef]

18. Lekkoksung, N.; Jampachon, P. Characterizations of regular ordered ternary semigroups in terms of fuzzy subsets. Southeast-Asian J. Sci. 2013, 2, 141-153.

19. Lee, K.J. Bipolar-vaued fuzzy sets and their operations. In Proceedings of the International Conference on Intelligent Technologies, Bangkok, Thailand, 13-15 December 2000; pp. 307-312.

20. Ibrar, M.; Khan, A.; Davvaz, B. Characterizations of regular ordered semigroups in terms of $(\alpha, \beta)$-bipolar fuzzy generalized bi-ideals. Int. J. Fuzzy Syst. 2017, 33, 365-376. [CrossRef]

21. Bashir, S.; Du, X. On Weakly Regular Fuzzy Ordered Ternary Semigroups. Appl. Math. Inf. Sci. 2016, 10, 2247-2254. [CrossRef]

22. Rehman, N.; Shabir, M. $(\bar{\alpha}, \bar{\beta})$-fuzzy ideals of ternary semigroups. World Appl. Sci. J. 2012, 17, $1736-1758$. [CrossRef]

23. Shabir, M.; Bano, M. Prime bi-ideals in ternary semigroups. Quasigroups Relat. Syst. 2008, 16, $239-256$.

24. Lekkoksung, N.; Jampachon, P. On regular ordered ternary semigroups. Far East J. Math. Sci. 2014, 92, 67-84.

(C) 2019 by the authors. Licensee MDPI, Basel, Switzerland. This article is an open access article distributed under the terms and conditions of the Creative Commons Attribution (CC BY) license (http://creativecommons.org/licenses/by/4.0/). 